\title{
tic\&société
}

Vol. 14, $\mathbf{N}^{\circ}$ 1-2 | 1er semestre 2020 - 2ème semestre 2020

Mutations numériques de la musique : des

contradictions à analyser

\section{La musique comme analyseur : mutations de la filière musicale et mutation de la recherche sur la musique}

Music as an analyser: mutations in musical sector and mutations in research on music

La música como analizador: mutaciones en el sector musical y mutaciones en la investigación musical

Christophe MAGIS et Lucien PERTICOZ

\section{(2) OpenEdition}

Journals

Édition électronique

URL : http://journals.openedition.org/ticetsociete/4666

DOI : 10.4000/ticetsociete.4666

Éditeur

Association ARTIC

Édition imprimée

Pagination : 13-65

Référence électronique

Christophe MAGIS et Lucien PERTICOZ, «La musique comme analyseur : mutations de la filière musicale et mutation de la recherche sur la musique », tic\&société [En ligne], Vol. 14, N 1-2 | 1er semestre 2020 - 2ème semestre 2020, mis en ligne le 11 novembre 2020, consulté le 23 février 2021 URL : http://journals.openedition.org/ticetsociete/4666; DOI : https://doi.org/10.4000/ticetsociete. 4666 
tic\&société - 14(1-2), 2020

\section{La musique comme analyseur : mutations de la filière musicale et mutation de la recherche sur la musique}

\section{Christophe MAGIS}

Christophe Magis est maître de conférences en sciences de l'information et de la communication à l'université Paris 8 où il est chercheur au Centre d'études sur les médias, les technologies et l'internationalisation (Cemti). S'inscrivant à l'articulation de l'économie politique de la communication et de la Théorie critique, ses recherches portent sur les mutations des industries culturelles. Sur la musique, il a notamment publié l'ouvrage La musique et la publicité. Les logiques socioéconomiques et musicales des mutations des industries culturelles (Paris, Mare et Martin, 2015). christophe.magis@univ-paris8.fr

\section{Lucien PERTICOZ}

Lucien Perticoz est enseignant-chercheur en sciences de l'information et de la communication à l'université Jean Moulin Lyon 3 et membre de l'équipe pluridisciplinaire MARGE (E.A. 3712). Ses travaux se positionnent au croisement de l'étude des évolutions des pratiques culturelles et d'une approche critique des mutations récentes des industries de la culture et des médias. II a dirigé, avec Jacob Matthews, l'ouvrage collectif L'industrie musicale à l'aube du XXI siècle. Approches critiques (2012). lucien.perticoz@univ-lyon3.fr 
La musique comme analyseur : mutations de la filière musicale et mutation de la recherche sur la musique

\title{
La musique comme analyseur : mutations de la filière musicale et mutation de la recherche sur la musique
}

Résumé : Première des industries culturelles à avoir subi les conséquences de son manque de préparation aux « révolutions numériques ", et notamment au succès de l'informatique connectée et de la norme MP3, l'industrie musicale a connu, au tournant du XXlème siècle, l'une des plus importantes crises de son histoire qui en est pourtant coutumière. Les vingt dernières années ont donc été celles à la fois d'une importante expérimentation sur le plan tant technique que socioéconomique et d'une documentation foisonnante quant aux perspectives de sortie de crise, ou aux transformations des usages sociaux de la musique. Cet article en propose une mise en perspective critique.

Mots-clés : industrie musicale, filière phonographique, numérique, pratiques, approche critique.

\section{Music as an analyser: mutations in musical sector and mutations in research on music}

\begin{abstract}
The first cultural industry to suffer from a lack of preparation for "digital revolutions" (e.g. the success of streaming and the MP3 standard), the music industry experienced one of the most important in a long series of crises at the turn of the $21^{\text {st }}$ century. The last twenty years have been characterized by major technical and socio-economic experiments, as well as an abundant literature on economic perspectives and transformations in the social uses of music. This article offers a critical perspective on these mutations.
\end{abstract}

Keywords: music industry, record industry, digital, practices, critical approach. 


\title{
Music La música como analizador: mutaciones en el sector musical y mutaciones en la investigación musical
}

\begin{abstract}
Resumen: La industria musical es la primera industria cultural que sufrió las consecuencias de su falta de preparación para las "revoluciones digitales", y en particular frente al éxito de los ordenadores conectados y del estándar MP3. Al principio del siglo XXI, la industria de la música experimentó una de las crisis más importantes de su historia. Los últimos veinte años han permitido una experimentación en los planos técnico y socioeconómico sin precedentes. Durante este periodo, se ha generado una abundante literatura sobre las perspectivas de salida de la crisis, y sobre las transformaciones de los usos sociales de la música. El objetivo de este artículo es proponer un análisis crítico de estas mutaciones.
\end{abstract}

Palabras claves: industria musical, sector fonográfico, digital, prácticas, enfoque crítico. 
La musique comme analyseur : mutations de la filière musicale et mutation de la recherche sur la musique

Si les travaux sur les mutations des industries culturelles nous apprennent combien celles-ci sont souvent à l'avant-garde des mutations plus générales du capitalisme (Bouquillion, 2008 ; Miège, 2007 ; Hesmondhalgh, 2013), force est de constater qu'en leur sein, la filière musicale est généralement la première annonciatrice des évolutions. La reconnaissance du droit d'auteur et la mise sur pied d'un puissant organisme de collecte et de répartition des droits, de laquelle la production musicale a été partie prenante et qui a donné le la des dispositifs de propriété intellectuelle du XXème siècle, ou encore l'importance prise par les sous-cultures dans le renouvellement des esthétiques et des codes sociaux et culturels, pour laquelle la production musicale a été à l'avant-poste des transformations sociales, sont révélatrices du rôle de premier plan que cette filière joue dans la construction et dans les transformations économiques, politiques et culturelles des sociétés.

Ce qu'on a pour habitude d'appeler l'« industrie de la musique $»-$ en la réduisant généralement malencontreusement à la seule vente de phonogrammes (Williamson et Cloonan, 2007) - prend son envol à la toute fin du XIXème siècle, et surtout à la Belle époque, à la suite d'un ensemble d'évolutions technologiques ayant permis l'enregistrement et la reproduction sonore (Tournès, 2011). Les usages associés aux appareils permettant d'enregistrer et de lire les sons ne se stabilisent qu'en participant au premier chef de l'essor de la culture de masse dans le loisir citadin du début du $X X^{\text {ème }}$ siècle et surtout de l'entre-deux-guerres ${ }^{1}$. La consommation de musique sur support disque est ensuite un élément essentiel de la « civilisation du loisir» (Dumazedier, 1962) associée à la " société de consommation » (Baudrillard, 1970). Fixée, par analogie avec la vente de partitions, autour d'un " éditeur » qui tire son nom de cette analogie même (Szendy, 2001), l'économie de la filière est connue pour sa sensibilité aux crises, dans lesquelles elle trouve néanmoins toujours à

1 On sait notamment que les premières «machines parlantes » ont été commercialisées dans un but de restitution de la voix parlée en vue d'usages proches de la photographie d'un côté (mémoire de la parole) et du dictaphone de l'autre. Thomas Edison, créant à cette fin la première société commerciale de vente de phonographes, écrit ainsi : "La principale utilité du phonographe est de permettre d'écrire des lettres, de dicter des textes. C'est dans ce but qu'il a été construit » (cité dans Flichy, 1997, p. 95). 


\section{Christophe MAGIS et Lucien PERTICOZ}

renouveler ses esthétiques, ses usages et ses modèles économiques (Vandiedonck, 2007). La dernière crise en date, au tournant du XXI ${ }^{\text {ème }}$ siècle, qui a vu, par exemple, en France, le marché perdre la moitié de sa valeur entre 2002 et 2007, a été très grandement documentée au début des années 2000 et au début des années 2010 dans un contexte d'incertitude quant à la reprise. Ainsi, sur le marché hexagonal, celle-ci n'intervient timidement qu'à partir du milieu des années 2010 grâce à la croissance des revenus numériques ${ }^{2}$ (figure 1 ). Cette crise a été l'occasion de transformations majeures, tant culturelles que sociales, politiques et économiques ainsi que scientifiques, que cette introduction voudrait documenter à partir d'un état de la littérature des vingt dernières années, en posant cette proposition comme hypothèse de départ : la musique comme analyseur.

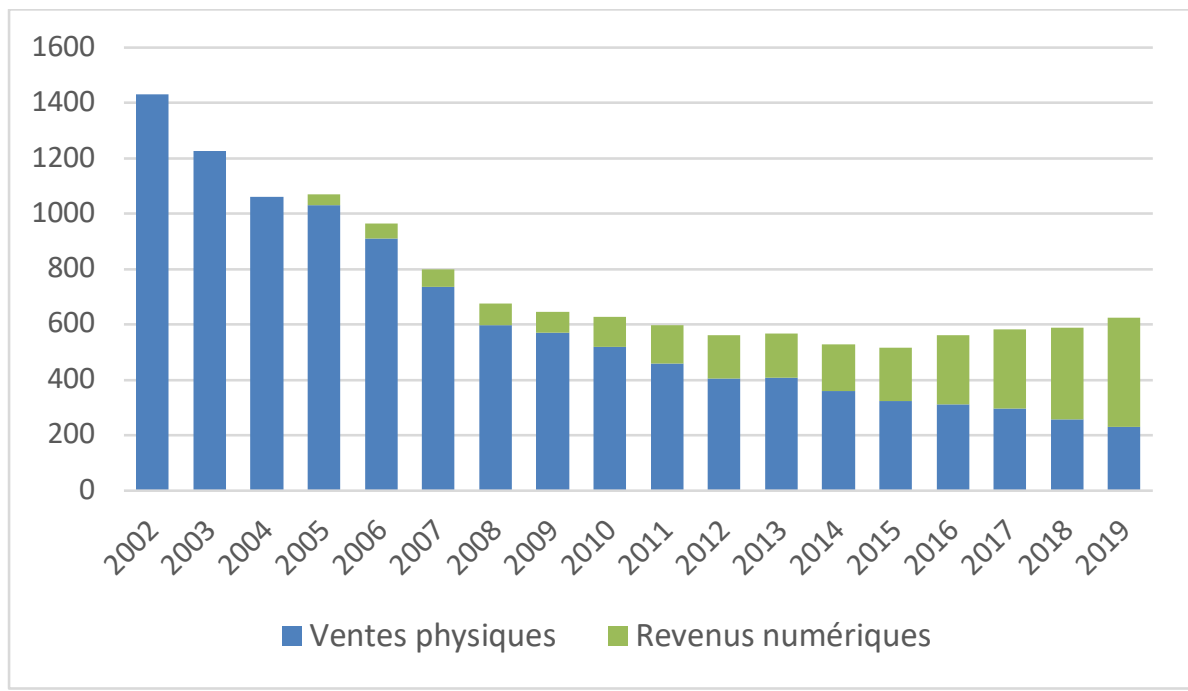

Figure 1. Évolution du chiffre d'affaires de la musique enregistrée en France, entre 2002 et 2019 (en millions d'euros - hors DV et synchronisation) (source : SNEP - La production musicale française 2019)

\footnotetext{
2 II convient à cet égard de souligner que, contrairement à celles communiquées par I'IFPI (International Federation of the Phonographic Industry), les données de marché du SNEP (Syndicat national de l'édition phonographique) ne font pas, semble-t-il, apparaître les revenus générés par les droits voisins et la synchronisation, ce qui viendrait peut-être nuancer le caractère " timide » de ladite reprise.
} 
La musique comme analyseur : mutations de la filière musicale et mutation de la recherche sur la musique

\section{Crises de la filière phonographique et mutations du capitalisme : la nécessité renouvelée d'une approche critique}

Comme pour bien d'autres filières des industries culturelles, mais peut-être encore davantage qu'elles, si l'on pense au livre ou au cinéma, les vingt dernières années ont été le théâtre d'une transformation importante de l'économie de la filière musicale et des représentations qui lui sont associées. Quiconque a vécu sa jeunesse durant les années 2000 des sociétés occidentales peut aujourd'hui regarder cette période en se rappelant à quel point la tentative, pour les majors du disque, de conserver leur fonctionnement économique qui s'était articulé depuis environ un siècle autour de la vente à l'unité de supports enregistrés a conduit à une inflation de discours sur le piratage lié au téléchargement illégal, un péril qui menacerait l'industrie, les artistes et la création artistique elle-même dans son entier ${ }^{3}$, alors que la contrefaçon de disques et de cassettes représentait pourtant dès 1990 un quart des phonogrammes vendus dans le monde (Curien et Moreau, 2006).

$\mathrm{Si}$, une vingtaine d'années plus tard, la question du piratage n'est plus au centre des imaginaires associés à l'industrie musicale, il n'en demeure pas moins que ces discours ont eu une place importante dans la préparation d'un certain nombre de cadres législatifs réglementant la circulation des productions musicales sur le web. Pourtant, il semble que les travaux sur l'économie de la musique, nombreux dans les années 2000 au plus fort de la crise et se sentant alors quasiment obligés de se situer par rapport à la question du piratage, ont été moins nombreux par la suite. II nous apparaît toutefois que c'est justement maintenant, tandis que l'industrie paraît amorcer une nouvelle phase de croissance, que de nouveaux travaux sur l'économie de la musique seraient nécessaires, susceptibles de replacer ces derniers cycles de crise-croissance dans la marche d'ensemble du capitalisme.

3 Situation d'emballement généralisé que le célèbre artiste satirique "Weird Al" Yankovic a parodié dans un pastiche hilarant du genre " charity song " (cf. "Weird Al" Yankovic, « Don't Download This Song » (3'54), album : Straight Outta Lynwood, Volcano Records, 2006). 


\section{Christophe MAGIS et Lucien PERTICOZ}

\section{1 « Crise du disque » et transformation de la filière}

L'économie de la filière phonographique a donc été au centre de bon nombre de travaux issus du monde scientifique, ainsi que de rapports professionnels commandités par tel ou tel groupe d'acteurs du secteur, et ce, dès la fin des années 1990. La "crise du disque ", qui a constitué une sorte de fil rouge des années 2000, tant pour les acteurs historiques de la filière que pour les chercheurs qui l'ont étudiée, a ainsi fait l'objet d'une grande documentation et d'une certaine publicité dans l'espace public, vue comme annonciatrice possible et sans précédent des changements dans les autres industries culturelles. Toutefois, s'il est une spécificité de l'industrie phonographique qui a également déjà été fort bien documentée, c'est celle de son histoire, rythmée par les crises récurrentes. Qu'il s'agisse, dans les années 1920, de la crise provoquée par le développement des offres radiophoniques, qui a eu comme conséquence une réorganisation du secteur autour d'acteurs issus de l'industrie radiophonique, qu'il s'agisse des crises provoquées par les essoufflements esthétiques des années 1950 et 1960, qui ont entraîné la stabilisation de la division du travail entre les acteurs dans la structuration du secteur en "oligopole à frange concurrentielle », chargeant les indépendants en particulier du rôle de découvreurs des esthétiques nouvelles (Hennion, 1981) ou qu'il s'agisse, au début des années 1980, de la crise consécutive à la fin du support vinyle qui a vu l'augmentation de la qualité technique de la reproduction audiophile avec l'imposition du format $C D$, les crises viennent régulièrement rebattre les cartes au sein de la filière, permettant l'arrivée de nouveaux entrants davantage en mesure de tirer profit de la nouvelle donne, ainsi que le rappellent Bourreau et LabarthePiol (2004) au cœur de cette crise des années 2000 :

L'apparition d'une nouvelle technologie peut entraîner une rupture dans la croissance des ventes. Elle peut conduire aussi à une réorganisation du secteur, avec l'entrée de nouveaux acteurs plus à même d'exploiter la nouvelle technologie. La numérisation de la musique devrait produire de tels effets (p. 51-52).

Sur ce dernier point, les données les plus récentes permettant de suivre l'évolution du chiffre d'affaires français et mondial de la filière leur donnent pleinement raison, ne serait- 
La musique comme analyseur : mutations de la filière musicale et mutation de la recherche sur la musique

ce que pour les seuls secteurs associés à la production/distribution musicale. La baisse des revenus s'est effectivement poursuivie jusqu'en 2014 (soit pendant encore 10 ans après la publication de leur article), mais force est de constater que, depuis, la musique enregistrée s'inscrit à nouveau dans un cycle de croissance à l'échelle mondiale. En 2019, les revenus cumulés ont ainsi quasiment retrouvé leur niveau de 2004, pour atteindre un chiffre d'affaires de 19,1 milliards de dollars US (figure 2). En revanche, certaines des prévisions des auteurs - qui ont peut-être sous-estimé la puissance des logiques propres à la valorisation du capital qui ne peut notamment faire l'impasse sur la distribution pour se réaliser - ne correspondent pas aux dynamiques observables à ce jour. En toute rigueur, le passage de la musique en régime numérique n'a ni amené « la quasi-disparition de la fonction de distribution, qui conférait un avantage concurrentiel aux grandes maisons de disques, les majors ", ni permis outre mesure de "stimuler la création musicale et l'entrée de nouveaux labels » (ibid.).

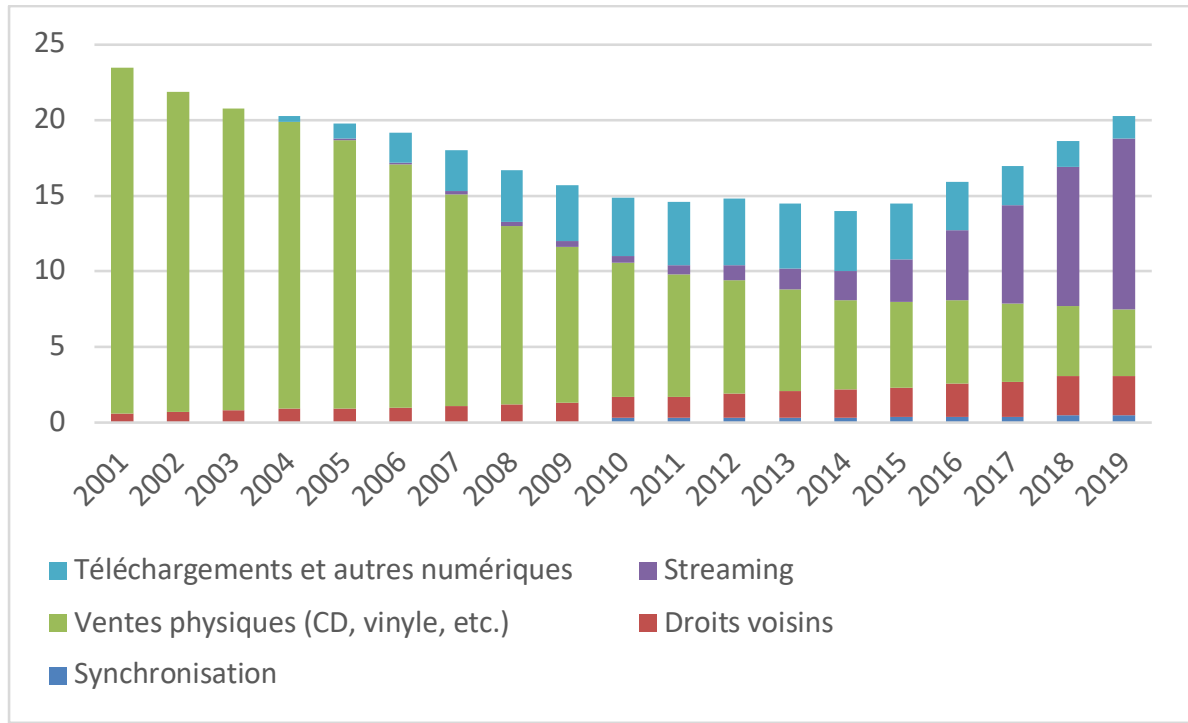

Figure 2. Évolution du chiffre d'affaires mondial de la musique enregistrée, entre 2001 et 2019 (en milliards de dollars US) (source : IFPI - Global Music Report - The Industry in 2019) 


\section{Christophe MAGIS et Lucien PERTICOZ}

Sur le premier aspect évoqué par les auteurs, les majors ont effectivement perdu une partie de l'avantage concurrentiel que leur procurait leur contrôle de la distribution musicale, mais cela n'est pas dû tant à la " quasi-disparition » de celle-ci qu'à son déplacement au profit de nouveaux entrants issus de l'industrie du numérique tels que Google, Apple ou Amazon (Bouquillion, 2008 ; Bouquillion, Miège et Mœglin, 2012 ; Gomez-Mejia Nicey et Vaezi, 2016 ; Gayraud et Heuguet, 2015). Concernant le second point, sa non-réalisation est, d'une certaine manière, liée au premier, car, face à l'irruption des acteurs du numérique à l'aval de la filière, les majors ont accéléré le processus de concentration du secteur de la production afin, notamment, de pouvoir faire valoir leur catalogue de droits auprès des plateformes numériques qui entendent les exploiter. En conséquence, les maisons de disques majors n'étaient plus que trois en 2019 (Universal Music Group, Sony Music Entertainment et Warner Music Group) $)^{4}$, tout en totalisant à elles seules $70 \%$ des revenus générés sur le marché de la musique enregistrée ${ }^{5}$.

D'une certaine manière, les auteurs cités ont eux-mêmes été contraints de constater que, malgré toutes les promesses supposées du numérique, le fonctionnement en oligopole ainsi que la domination de quelques acteurs étaient loin d'avoir disparu (Bourreau, Maillard et Moreau, 2015). Le numérique semble plutôt avoir contribué à un renforcement de ces logiques typiques de ce que Baran et Sweezy (1968) ont appelé le "capitalisme monopoliste " et sur lequel nous reviendrons infra.

Une fois ce constat posé (et accepté), il convient de pousser ce raisonnement jusqu'au bout de sa logique. Ainsi, le retour de la croissance du chiffre d'affaires de l'industrie phonographique entamé au mitan des années 2010 s'opère dans un contexte où les rapports de force entre acteurs économiques, s'ils ont pu favoriser l'arrivée de nouveaux entrants tels qu'Apple, Google/YouTube ou Spotify, n'ont pas entraîné une déconcentration du marché et une répartition plus équitable des

\footnotetext{
${ }^{4}$ Elles étaient encore quatre jusqu'en 2011 et cinq jusqu'en 2004.

${ }^{5}$ Richaud, N. (2019, 31 décembre). Musique enregistrée : Universal, Warner et Sony pèsent près de $70 \%$ du marché. LesEchos.fr. Repéré à https://www.lesechos.fr/techmedias/medias/musique-enregistree-universal-warner-et-sony-pesent-pres-de-70-dumarche-1000451
} 
La musique comme analyseur : mutations de la filière musicale et mutation de la recherche sur la musique

revenus. À cet égard, l'arrivée d'Apple sur le marché de la distribution musicale demeure un cas d'école. Rivées sur des discours associant le développement de la musique en ligne à du "piratage ", les majors ont laissé vacante la possibilité de proposer une offre légale de musique en ligne associée à de nouveaux usages. Lorsque la firme californienne a lancé l'iTunes Store en 2003, son but n'était alors pas tant de s'implanter sur le secteur musical que d'assurer une offre musicale compatible avec son baladeur numérique star, l'iPod dont le succès a grandement profité de sa capacité à lire également les titres pirates (Bouquillion, 2008). Compagnie puissante et extérieure au secteur, Apple, qui s'est rapidement taillé la " part du lion » du marché des baladeurs numériques portables (21,6 \% en 2003), a ainsi eu l'occasion de décider d'un prix type pour chaque titre à 0,99 \$US, sur lequel les offres légales issues des majors du disque qui lui ont emboîté le pas dans les années 2000 ont été contraintes de s'aligner.

Avec le développement des offres d'autres acteurs de l'oligopole des géants du web, nos propres conclusions formulées il y a de cela presque dix années (Matthews et Perticoz, 2012) semblent demeurer d'actualité : bien loin d'avoir entraîné une plus grande diversité des firmes positionnées sur la filière phonographique, la tendance reste à la domination économique d'une poignée d'entre elles. En d'autres termes, les mutations récentes de l'industrie musicale ont très rapidement permis de comprendre qu'Internet et le numérique, plutôt que de favoriser une rupture avec les logiques du capitalisme monopoliste, ont davantage contribué à les renforcer, quitte à faire émerger de nouveaux oligopoles, que ce soit autour des majors de l'édition phonographique ou des GAFAM.

En effet, forts de cette dynamique de croissance retrouvée, les « contenus » musicaux suscitent à nouveau l'intérêt des investisseurs, à tel point qu'Universal Music se voit maintenant qualifiée de «pépite » par la presse économique ${ }^{6}$. L'utilisation de ce vocable - qui est généralement plutôt réservé aux entreprises de l'économie numérique au plus fort potentiel de

${ }^{6}$ Richaud, N. (2019, 17 octobre). Après Tencent, Vivendi va poursuivre l'ouverture du capital de sa pépite Universal Music. LesEchos.fr. Repéré à https://www.lesechos.fr/tech-medias/medias/apres-tencent-vivendi-va-poursuivrelouverture-du-capital-de-sa-pepite-universal-music-1140989 


\section{Christophe MAGIS et Lucien PERTICOZ}

croissance supposé - évoque d'une certaine manière le basculement de la filière phonographique dans une économie dite "de plateforme" dont elle a contribué à la montée en puissance, tout en dépendant de plus en plus économiquement de celle-ci. À cet égard, il convient de souligner que la crise des années 2000 et la « révolution numérique » n'ont pas conduit la production musicale à sortir des logiques de valorisation du capital: elle semble au contraire avoir confirmé son positionnement à l'avant-garde de ses évolutions les plus récentes, surtout si l'on songe à l'intérêt que lui portent les GAFAM ou Tencent en Chine ${ }^{7}$. II nous semble que ces évolutions récentes illustrent de manière assez exemplaire comment l'innovation technique, tout en étant présentée dans les discours qui l'accompagnent comme le moyen d'extraire les productions humaines des logiques de valorisation du capital, contribue bien au contraire à en accroître l'emprise, comme l'avait notamment pressenti Jacob Matthews (2012) lorsqu'il estimait, au tournant des années 2010, que « la musique [était] en passe d'achever sa transformation en une activité exclusivement orientée vers l'accumulation capitaliste » (p. 17).

En effet, la concentration par fusion/acquisition observée au sein de la filière phonographique à la suite de ladite "crise du disque " - qui a abouti, comme nous le rappelions, à un resserrement du marché autour de seulement trois majors - ne doit pas faire oublier l'arrivée de puissants nouveaux acteurs qu'Internet et le numérique ont précisément rendu possible, ni combien ces nouveaux acteurs, arrivant avec leurs organisations et leur fonctionnement économiques propres, sont également au cœur de la transformation des logiques marchandes et de leur articulation autour de l'infomédiation: «la logique centrale et particulière de l'oligopole [que représentent les GAFAM] est sa volonté d'organiser les deux activités génériques de l'internet, c'est-à-dire la communication interpersonnelle et la diffusion de contenus sur supports numériques ainsi que toute autre activité se trouvant à leur confluence »(Smyrnaios, 2017, p. 71).

\footnotetext{
${ }^{7}$ Madelaine, N. (2019, 31 décembre). « Vivendi signe avec Tencent pour l'ouverture du capital d'Universal Music. LesEchos.fr ». Repéré à https://www.lesechos.fr/techmedias/medias/vivendi-signe-avec-tencent-pour-louverture-du-capital-duniversalmusic-1159662 (consulté le 26 juin 2020).
} 
La musique comme analyseur : mutations de la filière musicale et mutation de la recherche sur la musique

L'industrie musicale a été - malgré elle - l'une des premières à fournir à profusion ces "contenus musicaux », mais sans réussir tout à fait à en contrôler ni la reproduction ni la distribution. L'arrivée tardive des majors sur le segment de la distribution légale a quelque peu laissé la place à ces acteurs nouveaux et autrement plus solides économiquement qui ont pu utiliser la musique (ainsi que d'autres productions culturelles) comme «produit d'appel» permettant le développement de leurs offres et l'affinement de leurs modèles économiques, à l'écart des routines de travail de la production musicale (Bouquillion, Miège et Mœglin, 2013).

\subsection{Crises, discours de crises et discours sur la crise : pour une approche critique}

Comme pour les autres objets de la culture et de la communication, les transformations de l'industrie musicale sont régulièrement susceptibles de faire l'objet de discours, tantôt alarmistes, tantôt iréniques, notamment en ce qui concerne l'évolution économique.

D'un côté, en effet, les discours de crise émanant des cadres du secteur de la production comme de la distribution phonographique et centrés sur la notion de piratage ou de piraterie, par laquelle l'industrie a réussi ce tour de force de présenter ses propres clients comme responsables de ses malheurs économiques sont encore dans les mémoires ${ }^{8}$, tout comme les actions de justice et les rappels à la loi visant à faire clairement savoir à quel point ce délit était grave! À titre d'exemple, l'implémentation en France de la directive européenne 2001/29/CE sur l'harmonisation des droits d'auteur et des droits voisins dans la société de l'information (qui avait été ratifiée après force lobbying de la part entre autres des industriels de la musique) a résulté en l'adoption avec éclat en

8 Et notamment les mots de Pascal Nègre, alors président-directeur général d'Universal Music France, selon lesquels «la piraterie c'est du vol » ainsi que ses propos contre le principe de "licence globale »... qui sera finalement, avec le streaming, le modèle plébiscité in fine. Cf., par exemple: Poussielgue, G. (2003, 17 janvier). Interview Pascal Nègre : « Les FAl doivent empêcher l'accès aux sites pirates ", Lesechos.fr. Repéré à https://www.lesechos.fr/2003/01/pascal-negre-lesfai-doivent-empecher-lacces-aux-sites-pirates-657954; Dunand, C. (2008, 24 avril) Interview Pascal Nègre: "quel est l'avenir de la création sur internet ?", Lesechos.fr. Repéré à https://www.lesechos.fr/2009/04/pascal-negre-quel-estlavenir-de-la-creation-sur-internet-473526. 


\section{Christophe MAGIS et Lucien PERTICOZ}

2006 de la loi dite "DADVSI », malgré une forte opposition de la part d'associations de consommateurs et d'associations professionnelles alertant sur son caractère particulièrement restrictif et liberticide (Battisti, 2004 ; Schöpfel, 2006). Cette adoption est allée de pair avec plusieurs campagnes de prévention au ton très paternaliste et moralisateur.

De l'autre, de manière symétrique, la décennie 2000 a été celle d'un engouement régulièrement renouvelé pour l'impact majeur que devrait ou aurait dû apporter tel nouvel outil technique ou telle nouvelle proposition économique. Lors d'une journée d'étude organisée à la MSH Paris-Nord en janvier 2010 dans le cadre du programme de recherche "L'industrie musicale à l'aube du XXI ${ }^{\text {ème }}$ siècle » piloté par Jacob Matthews et auquel nous participions, plusieurs intervenants professionnels s'emballaient alors en présentant certaines de ces propositions. À l'époque, c'était notamment le crowdfunding qui avait le vent en poupe autour du label MyMajorCompany et de son artiste phare, Grégoire, et qui était censé être la nouvelle manière de produire de la musique, c'est-à-dire moins centralisée que dans le cadre des industries culturelles traditionnelles, selon la rhétorique d'alors autour du Web collaboratif (Bouquillion et Matthews, 2010). Tel acteur nous présentait par ailleurs ce qui était censé devenir un succès "révolutionnant à la fois la production et l'expérience musicale ", l'outil MXP4... qui n'a pas rencontré le succès escompté et dont plus personne ne parle ${ }^{9}$. Tel autre avançait encore un discours alors répandu selon lequel c'était par des partenariats avec les marques que les artistes allaient désormais continuer à vivre de leur musique, à l'extérieur du contrôle des maisons de disques, et notamment des majors. Elles sont pourtant toujours présentes ${ }^{10}$ !

Si les discours se sont aujourd'hui adoucis, il ne faudrait pourtant pas oublier combien cette question du piratage, associée à l'époque à celle du terrorisme sur la liste des maux

\footnotetext{
9 L'engouement était alors largement partagé dans le petit milieu musical (cf., par exemple: Youngs, I. (2010, 28 janvier). Shrewd stars make music add up, BBCNews. Repéré à http://news.bbc.co.uk/2/hi/entertainment/8483665.stm).

${ }^{10}$ Comme le remarque d'ailleurs Jean-Philippe Louis (2020, 4 juillet). Non, les majors du disque ne sont pas mortes. Lesechos.fr. Repéré à https://www.lesechos.fr/2018/07/non-les-majors-du-disque-ne-sont-pas-mortes998023. Et elles ne se sont, pour cela, peut-être pas tant « réinventées » que ce que semble penser l'auteur.
} 
La musique comme analyseur : mutations de la filière musicale et mutation de la recherche sur la musique

des sociétés occidentales (Vandiedonck, 2007), en est venue, dans les mesures qui ont été prises par les États et les institutions supranationales pour tenter de l'enrayer, à donner le la à une grande partie des règles qui régentent aujourd'hui l'Internet. Autrement dit, il ne faudrait pas sous-estimer son rôle dans ce qu'Armand Mattelart (2007) appelle « la globalisation de la surveillance ». La nécessité d'approcher la filière musicale au sein des recherches sur les industries culturelles, à partir d'une économie politique de la communication, nous semble ainsi évidente, à la suite de Matthews (2012). Approche critique majeure des études de communication, s'appuyant en grande partie sur des cadres critiques issus du marxisme occidental (Granjon, Guyot et Magis, 2019), l'économie politique de la communication a pour ambition générale de penser les transformations des structures de production de la culture, de l'information et de la communication en rapport à l'évolution générale du capitalisme. Elle entend ainsi mettre en rapport les mutations socioéconomiques avec les discours qui les accompagnent et les justifient, qu'ils soient d'ordre politique, technique ou scientifique.

Dans le monde francophone, cette approche a notamment été investie dans le cadre de ladite "théorie des industries culturelles », centrée autour des figures de Bernard Miège en France (Magis, 2019) et de Gaëtan Tremblay au Québec (George, 2014). Cette tradition a entre autres permis la mise en place d'une réflexion critique sur les modalités de structuration des filières autour des logiques mises en place par un acteur central. Le célèbre chapitre sur "Le disque " de l'ouvrage Capitalisme et industries culturelles (Huet et al., 1978 - souvent présenté comme le point de départ de la tradition de recherche francophone sur les industries culturelles) propose ainsi une critique fine du dispositif du droit d'auteur dans les modalités d'exploitation de la force de travail dans l'industrie musicale. Là où d'autres propositions d'analyse de l'économie de la musique comme celle de Jacques Attali (1977, p. 80 sq.) voyaient en ce dispositif une forme d'émancipation d'un type de travail créateur par l'assimilation de sa rémunération à une rente, l'économie politique de la communication montre combien, en réalité, le droit d'auteur permet au capital engagé dans la production musicale de ne s'acquitter du paiement du travail de création qu'en cas de succès et au prorata des volumes de ventes soit, en réalité, de ne pas payer l'essentiel du travail de 


\section{Christophe MAGIS et Lucien PERTICOZ}

création, les succès étant bien plus rares que les échecs. Autrement dit, les droits d'auteurs, ainsi qu'un certain nombre de systèmes de rémunération dérivés comme les royalties, permettent de « laisser à la charge des artistes la plus grande part des risques liés au caractère particulièrement aléatoire du processus de valorisation" (Huet et al., 1978, p. 97). C'est ainsi que peut s'expliquer le maintien d'un système de rémunération d'aspect si archaïque, au sein même du mode de production capitaliste, qui, s'articulant sur l'exploitation de la force de travail au-delà de ce qu'elle est effectivement rémunérée, voit traditionnellement dans le salariat son mode de rémunération de prédilection. Par ailleurs, les discours et les imaginaires associés au dispositif de droit d'auteur permettent d'associer les productions ainsi rémunérées à une « figure » de l'artiste et du travail artistique, ce qui permet un « maintien de l'aura des activités artistiques »: "Les marchandises culturelles, en effet, doivent continuer à être marquées du sceau sinon de l'unique, du moins du génie créatif, et ainsi apparaître, non comme émanant de laboratoires de recherche, mais comme conçues par des artistes ne dépendant pas d'industriels. " (Miège, 2017, p. 34.)

Cette réflexion sur les transformations du droit d'auteur dans la production musicale et de ses fonctions en parallèle des mutations plus générales du capitalisme mériterait d'être prolongée. Dans le monde francophone, une proposition en ce sens a été celle de Vincent Bullich (2011). À partir d'un état de la littérature sur l'intégration progressive des enjeux du droit d'auteur dans les travaux sur les industries culturelles, mais aussi de la littérature juridique sur les évolutions du droit d'auteur, Bullich (2011) propose de considérer conceptuellement ce dernier comme "mode de régulation", soit comme une " "forme institutionnelle" historiquement située, ni unique ni même nécessaire, mais qui contribue fortement à la structuration du mode de production et de consommation des marchandises culturelles au sein d'une économie capitaliste " (p. 57). Ainsi, dans une perspective inspirée de la théorie de la régulation, l'auteur montre l'importance majeure des droits dans les stratégies industrielles des acteurs de la production culturelle : ils permettent à la fois la valorisation d'un même contenu sur plusieurs médias en même temps qu'ils « constituent une modalité de gestion de la concurrence intra et intersectorielle » (p.63). Or, comme Bullich l'indique, peu de 
La musique comme analyseur : mutations de la filière musicale et mutation de la recherche sur la musique

travaux sont disponibles, notamment sur la musique, permettant d'évaluer précisément l'importance de la gestion des catalogues de droits dans les mutations des stratégies des industries culturelles. Nous ne pouvons qu'encourager avec lui de nouveaux travaux allant dans ce sens.

Par ailleurs, au-delà de la dimension d'étude stratégique des enjeux liés à la gestion des droits, il serait intéressant d'articuler une telle proposition avec celle émanant de travaux plus généraux sur la propriété intellectuelle et son évolution en rapport avec le capitalisme "des plateformes " (Vercellone, 2020), afin d'étudier comment cette évolution voit les créateurs et les musiciens, voire certains éditeurs phonographiques, réduits à l'état de producteurs de contenu pour des plateformes comme YouTube ou surtout Spotify, encouragés à toujours " s'engager davantage " $^{11}$. Et une telle articulation pourrait aussi intégrer une réflexion sur les usages qu'induisent ces nouvelles offres d'accès à la musique.

\section{2. Émancipation des auditeurs : promesses non tenues et réflexion sur les usages}

Comme le souligne Magaudda (2019), il apparaît que tout au long de l'histoire, une tendance nette se dégage, celle d'un "secteur musical [qui] se distingue par sa propension à adopter des nouvelles technologies numériques " (p.856), se positionnant ainsi à l'avant-garde des mutations qui traversent, depuis l'invention du disque compact en 1979, l'ensemble des filières des industries culturelles. Du point de vue des publics, ce passage au numérique des pratiques d'écoute musicale a été, jusqu'à très récemment, généralement présenté sous un jour favorable et comme un facteur de progrès pour les auditeurs. Ce point - voire ce tropisme - a notamment été souligné par Philippe Le Guern (2014) dans son état de l'art des articles traitant de la culture publiés dans la revue Réseaux depuis sa création. II conclut ainsi sur le fait que, dans la très grande majorité des contributions recensées, il ressort à leur

11 Cf., par exemple, le billet de Lelo Jimmy Batista sur le blogue culture de Libération: (2020, 25 septembre). Spotify, une pirouette pour des cacahuètes. Repéré à https://next.liberation.fr/musique/2020/09/25/spotify-une-pirouette-pourdes-cacahuetes 1800590?fbclid=IwAR3fKX9EvlUkV-

G9AxMtd4XqsNsTsug5Q9DknbYUA-1h KgUATZiYb66z3c 


\section{Christophe MAGIS et Lucien PERTICOZ}

lecture " [qu']avec le numérique et sa généralisation, l'idée selon laquelle nous assistons à une forme de progrès s'est largement répandue et semble faire l'objet d'un relatif consensus. Ce progrès est d'ailleurs entendu comme un progrès "démocratique" ou "démocratisant" » (p. 243).

L'auteur, au moment d'évoquer ce postulat de départ, parle d'une " pseudo-évidence » qu'il serait nécessaire de discuter. II nuance un certain nombre de lieux communs prenant pour acquis que le numérique, par ses spécificités techniques, favoriserait la remise en question des monopoles, contribuerait à une plus grande transparence dans la répartition des revenus, voire permettrait in fine une sortie de la culture du régime de la marchandise dans lequel elle est entrée au début du XXème siècle. Cette deuxième partie entend donc poursuivre cette discussion en insistant plus particulièrement sur le fait qu'au-delà d'un accès facilité à une profusion de contenus musicaux, le passage au numérique a surtout permis de documentariser les pratiques des auditeurs, rendant ainsi possible leur marchandisation (Morris, 2015a; Micheau, Després-Lonnet et Cotte, 2017). Dès lors, cette tendance, observée de manière très nette sur les plateformes de streaming musical, doit également nous interroger sur le type d'auditeur produit par celles-ci.

\subsection{Le numérique : entre " libération » de l'expérience musicale et dernier avatar de sa marchandisation}

Nombre de travaux visant à mettre au jour les implications culturelles, sociales et économiques de la numérisation de la musique se sont attachés, au cours des vingt dernières années, à essayer de rendre compte du potentiel « disruptif » d'Internet et du numérique, notamment dans leur capacité à favoriser une rupture avec le "vieux monde " industriel, hiérarchisé, concentré et aliénant qui caractériserait le capitalisme " d'avant ». Sans nécessairement s'inscrire dans le cadre d'une " utopie numérique » (Turner, 2012) dont Nicholas Negroponte, Henry Jenkins ou Richard Florida seraient les représentants les plus extrêmes, nombre de recherches des années 2000 ont pu formuler des problématiques visant à analyser comment les usages de la musique numérique - c'est-à-dire d'un support matériel spécifique d'inscription des messages - rendaient envisageable une rupture avec les organisations hiérarchiques 
La musique comme analyseur : mutations de la filière musicale et mutation de la recherche sur la musique

propres au capitalisme industriel. Sans aller jusqu'à ce scénario, certains auteurs (Bourreau, Gensollen et Moreau, 2007 ; Bourreau et Gensollen, 2006) ont pu estimer que ces évolutions étaient porteuses d'une potentielle remise en question du Star system et de " l'économie du hit » qui le soustend, considérant à cet égard que :

Lorsque les CD sont remplacés par des fichiers numériques, les coûts de production, dans une certaine mesure, et surtout les coûts de distribution, se réduisent fortement. De tels gains de productivité permettent une consommation plus diversifiée ainsi que le développement de pratiques intermédiaires entre la consommation et la production: les amateurs peuvent accéder à une qualité professionnelle pour les coûts limités d'un home studio. Les œuvres se désacralisent. Elles se réemploient, se composent avec d'autres, se réorganisent en œuvres nouvelles. Compilation, remix, sampling sortent des limites étroites du droit d'auteur comme les échanges sur les réseaux de pair à pair interrogent l'exception pour copie privée (Bourreau, Gensollen et Moreau, 2007, p. 15).

Au-delà d'une certaine vision de la technique, c'est également une image bien particulière de l'auditeur que ce passage donne à voir : celle d'un individu "créatif », en quête incessante de nouveautés et qui aurait été frustré par les restrictions d'usage des œuvres musicales induites par les évolutions de la réglementation en matière de droit d'auteur, ce qui ne va pas de soi quand on interroge les auditeurs sur leurs pratiques d'écoutes musicales (Perticoz, 2016). En d'autres termes, le numérique viendrait "libérer » l'auditeur en lui permettant de s'émanciper du joug des acteurs traditionnels de la filière - et plus précisément de celui des majors - tout en estimant que « non seulement la musique est consommée en plus grande quantité, mais elle est aussi mieux choisie et mieux adaptée aux goûts de chacun » (Bourreau, Gensollen et Moreau, 2007, p. 15). Ici, la capacité notamment des majors à " tirer parti de ce que le progrès technique, internet et la numérisation permettent » (ibid.) est mise en doute, considérant que «les consommateurs évoluent plus rapidement, [...] contournent les interdictions et inventent de nouveaux modèles " (ibid.). Internet et le numérique seraient donc porteurs d'une libération de la consommation de musique que 


\section{Christophe MAGIS et Lucien PERTICOZ}

les auditeurs auraient pleinement saisie, pendant que les majors restaient arc-boutées sur un modèle voué à disparaître, incapables d'appréhender le - et de tirer profit dudéplacement du « centre de gravité de la création de valeur [...] des contenus vers la méta-information nécessaire à leur consommation » (ibid.). À ce stade, les nouveaux acteurs tels que YouTube sont présentés comme davantage en mesure d'exploiter ces mutations, ce que la suite des événements viendra confirmer, mais sans que nous assistions pour autant à la disparition du star-system et de l'« économie du hit ».

Sur ce point, Nick Prior (2012) propose une contribution peut-être plus nuancée et, en tout état de cause, moins " essentialisante " dès lors qu'il s'agit d'appréhender les " effets" (économiques, culturels, artistiques, sociaux) de la numérisation des productions et des pratiques musicales. Dans le cadre de ses recherches sur le format MP3, l'auteur rappelle préalablement que certains acquis de la recherche en sciences humaines portant sur la technique n'ont pas été balayés par la radicalité supposée des supports numériques et insiste sur un point, parfois un peu oublié, à savoir que :

Le numérique n'est en fait pas qu'une technologie, mais un ensemble de significations, d'objets et de pratiques. II prend forme dans l'agencement d'un monde de plus en plus informatisé et interconnecté et se manifeste au quotidien au travers de nos comportements et de nos modes relationnels. Redisons-le, vouloir extraire l'objet technologique de ces modes relationnels et attribuer à la technologie un pouvoir indépendant, déconnecté de toute activité humaine, de tout savoir ou de toute structure sociale, c'est bel et bien commettre une erreur. Nous devons effectivement reconnaître que l'omniprésence de la technologie numérique est indissociable de l'essor d'une économie capitaliste de marché mondialisée, de plus en plus subordonnée à des pratiques de communication rapides (Prior, 2012, p. 70 ; nous soulignons).

Dès lors que le numérique est réenchâssé dans le maillage complexe des relations sociales, des rapports de production, des stratégies et des tactiques des acteurs des « mondes de l'art » qui participent de la dynamique de l'économie, de la création et des pratiques musicales, il apparaît à la fois comme 
La musique comme analyseur : mutations de la filière musicale et mutation de la recherche sur la musique

révélateur et partie prenante des logiques qui sous-tendent celles-ci. Ainsi, sans sous-estimer les mutations que la numérisation de la musique a rendues possibles, Prior (2012) souligne à juste titre « que [malgré celles-ci] le système du droit d'auteur au sein de l'industrie musicale traditionnelle, l'architecture des grands groupes de média et la logique capitaliste qui les sous-tend sont demeurés globalement en l'état, même s'ils ont dû faire quelques efforts d'adaptation » (p. 68). Autrement dit, si le passage au numérique a effectivement permis à de nouveaux entrants - tels Apple, Google ou Spotify pour les plus connus d'entre eux - de venir déstabiliser les positions acquises par les majors, la radicalité des mutations observées n'est pas allée jusqu'à remettre en question les logiques de valorisation marchande de la musique ainsi que celles de valorisation du capital. La musique envisagée sous sa forme numérique semble plutôt avoir élargi encore un peu plus les possibilités offertes à celle-ci.

C'est en ce sens que l'importante contribution de Sterne (2012 ; 2015 ; Sterne, Sklower et Heuguet, 2017) doit selon nous être envisagée. En effet, alors que l'auteur a proposé dans le cadre d'une approche épistémologique et méthodologique assez proche de celle de Prior - une analyse extrêmement fine du MP3 envisagé en tant qu'«artefact culturel » lui-même " forgé à la croisée de différentes industries électroniques, de l'industrie phonographique et des pratiques d'écoute attestées et idéales "(Sterne, 2015, p. 43), il nous semble que certaines conclusions auxquelles il parvient méritent une attention toute particulière. Sans le formuler de cette manière, Sterne montre ainsi de manière assez remarquable que le MP3 est un format qui s'inscrit pleinement dans le « nouvel esprit du capitalisme », tel qu'il a été décrit par Boltanski et Chiapello (1999). En effet, la « cité par projets » et la «critique artiste " viennent assez naturellement à l'esprit lorsque Sterne souligne que :

Le MP3 est une forme conçue pour l'échange massif, l'écoute informelle et l'accumulation massifiée. En tant que technologie-récipient pensée pour exécuter un processus sur les données qu'il contient, le MP3 accomplit les tâches pour lesquelles il a été programmé. Les usages primaires et illégaux du MP3 ne sont pas des usages aberrants ou des failles de la technologie ; ils constituent sa plus haute 


\section{Christophe MAGIS et Lucien PERTICOZ}

injonction morale: «Éliminez les redondances! Économisez de la bande passante! Voyagez tout le temps, voyagez loin, en un clin d'œil ! Entassez-vous dans les disques durs de la classe moyenne! Adressez-vous à un sujet auditeur distrait ! » (Sterne, 2015, p. 57-58; nous soulignons).

Dès lors, le numérique s'inscrit dans toute une histoire de la contre-culture des années 1960 telle qu'elle a été formalisée par Turner (2012). Bien loin de remettre en cause la propriété des moyens de production ainsi que les logiques de marchandisation et de valorisation qui sous-tendent le capitalisme, les différentes innovations techniques qui se sont succédé ont ouvert de nouvelles possibilités de valorisation du capital, non plus seulement sur les contenus, mais également sur les pratiques culturelles et sociales dont ils sont le support.

C'est précisément à cette lumière qu'il convient peut-être d'analyser l'attention accrue portée aux " méta-informations " (ou métadonnées) évoquées par Bourreau, Gensollen et Moreau (2007). Le passage au numérique n'aurait dès lors pas tant abouti à une libération de la consommation musicale qu'à la création de nouveaux marchés ainsi qu'à la marchandisation de pratiques qui n'avaient auparavant aucune valeur d'échange. C'est cette lecture que propose notamment Morris (2015a) en apportant une contribution en forme de contre-pied où il démontre, de manière fort convaincante, que la montée en puissance du numérique, loin d'émanciper la musique de son statut de bien marchand, a au contraire poussé encore un peu plus loin le processus de sa marchandisation. II estime ainsi qu'Apple peut être considéré comme le premier acteur à avoir tiré profit des particularités de ce qu'il nomme la « marchandise musicale numérique ». En élargissant - et en rentabilisant - le processus de marchandisation à l'ensemble de l'expérience musicale de l'auditeur, Apple ambitionnait d'intégrer dans l'ensemble de ses offres ce que l'auteur nomme la "gestion des modes de vie numériques " (Morris, 2015a, p. 145), c'està-dire une marchandisation de l'ensemble des expériences liées à l'écoute de musique numérique. Les offres d'écoute de streaming qui suivront à partir de 2008 (année du lancement de Spotify) accentueront cette tendance en plaçant l'auditeur au centre «d'un réseau de technologies et d'un processus de 
La musique comme analyseur : mutations de la filière musicale et mutation de la recherche sur la musique

marchandisation constante de l'expérience musicale " (ibid., p. 169).

Dès lors, en documentant la pratique même consistant à écouter la musique (Quel morceau ? Dans quel contexte ? Avec quel appareil de lecture? Etc.), le numérique a ainsi facilité l'entrée de celle-ci dans la sphère des activités dites " productives »; par conséquent, le numérique est ici envisagé comme l'instrument de la transformation de cette pratique en une marchandise, c'est-à-dire en " une médiation sociale spécifique au capitalisme " (Ouellet, 2016, p. 50). Cette marchandisation de la pratique même, que le passage au numérique a rendu possible, est ainsi au cœur des questions sur la place et le rôle de l'auditeur au sein des plateformes de streaming, dernier avatar de la "numérimorphose " des pratiques d'écoute musicale (Granjon et Combes, 2007).

\subsection{Quel auditeur pour quelles pratiques d'écoute en régime numérique?}

Dans leur article de 2007, Granjon et Combes concluent ainsi leur contribution sur une hypothèse forte, à savoir que :

Ce [qui est] désigné par numérimorphose peut-il être saisi comme le signe, dans le champ de la production/réception musicale, du développement d'un certain individualisme de type expressif. Celui-ci, s'appuyant sur les technologiques numériques les plus récentes, produirait de nouvelles formes d'amateurisme qui, renouvelant les prises de l'amour de la musique, permettraient que s'expriment des formes consommatoires dont l'objet serait finalement moins la musique que l'amateur lui-même (p. 331 ; nous soulignons).

Ils soulignent à cet égard que «les pratiques de consommation musicale sont par exemple de plus en plus diversifiées et s'accompagnent d'un déplacement des signes de distinction de la nature des contenus eux-mêmes vers les modalités de consommation de ceux-ci » (ibid.), c'est-à-dire d'une attention plus grande portée au $(\mathrm{x})$ moment(s) où la pratique se réalise effectivement. L'hypothèse sous-jacente ici serait que, dans des environnements de plus en plus « musicalisés » (Bull, 2000, 2007 ; Nowak, 2013 ; Rouzé, 2005, 2010), voire saturés de musiques et de contenus musicaux, la 


\section{Christophe MAGIS et Lucien PERTICOZ}

production de la valeur ne serait plus tant située du côté de ceux-ci - et donc des éditeurs qui les produisent - que de celui de leurs réceptions par les auditeurs que les plateformes de streaming telles que Spotify ou YouTube entendent capter et valoriser (Perticoz, 2012a, 2012b). La numérisation de la musique aurait ainsi contribué à renforcer une tendance déjà observée par Bull (2000) avec la multiplication des dispositifs d'écoute, l'auteur suggérant notamment que «[c]'est le quotidien qui est maintenant ré-auratisé, et ce, précisément grâce aux techniques de reproduction mécanique. [...] Phénoménologiquement, les usagers de systèmes stéréo personnels font l'expérience d'une vie de tous les jours potentiellement auratique ${ }^{12} »($ p. 132).

Ce déplacement de l'aura - et donc de la valeur - du côté de la réception amène dès lors à se questionner sur le statut de l'auditeur dans le processus même de valorisation du capital, c'est-à-dire non seulement du rôle qu'il y joue, mais également dans les modalités sociales, économiques et techniques qui fondent le devenir marchand de ses pratiques d'écoute musicale quotidiennes. Ce mouvement a ainsi amené certains chercheurs à porter leur attention - dans le cadre d'approches quelquefois davantage compréhensives que critiques - sur les pratiques en tant que telles, que ce soit pour mettre au jour les manières dont la musique est mobilisée par les individus pour gérer leurs affects et se construire une (des) identité(s) (DeNora, 2000, 2001; Frith, 1996) ou pour « modeler » leur environnement sonore par leurs différents usages des technologies numériques mises à leur disposition (Nowak, 2013, 2016). Dans la lignée des recherches d'Hennion (2004, 2007, 2009) sur les amateurs de musique et la réflexivité de leurs pratiques, ces travaux s'attachent essentiellement à mettre en avant "l'autonomie d'un amateur qui serait nécessairement un usager actif, inventif, accompli et entrepreneur de sa propre passion " (Granjon et Combes, 2007, p. 331), point sur lequel nous reviendrons. Par ailleurs, si les dispositifs numériques, en tête desquels les plateformes de streaming figurent en bonne place, sont envisagés comme des moyens supplémentaires de réaliser sa pratique, notamment en

\footnotetext{
12 Traduction libre de : "[lt is] experience [that] is now re-auratized precisely through forms of mechanical reproduction. [...] Phenomenologically, personal-stereo users experience daily life as potentially auratic. »
} 
La musique comme analyseur : mutations de la filière musicale et mutation de la recherche sur la musique

termes de possibilités renouvelées de découvertes musicales, ces travaux entendent à raison ne pas essentialiser le " numérique ». En effet, le principal mérite de ces recherches relevant d'une sociologie pragmatique est d'interroger les " effets " du numérique à la lumière des pratiques, des représentations et des valeurs préexistantes, c'est-à-dire en soulignant notamment « la persistance de certains dispositifs, arts de faire et dispositions en matière de découverte musicale " (Maisonneuve, 2019, p. 72). Ce faisant, c'est la capacité des auditeurs, en régime numérique, à construire de manière autonome leurs goûts et leurs répertoires musicaux qui est ici affirmée, notamment face à la montée en puissance des algorithmes de recommandation.

En effet, la question de la figure de l'auditeur qui émerge dans et par l'usage des plateformes d'écoute de musique en streaming ainsi que de la marge de manœuvre qui lui est laissée au sein de ces dispositifs est au cœur de nombre de recherches les plus récentes concernant les mutations des consommations musicales en régime numérique (Bouquillion, 2020 ; Beuscart, Coavoux et Maillard, 2019 ; Dupuy-Salle, 2019 ; Gilliotte, 2019 ; Gras, 2014, 2015 ; Le Guern, 2016 ; Micheau, Després-Lonnet et Cotte, 2017 ; Morris, 2015b). Ainsi, dans le prolongement théorique des travaux mentionnés précédemment, Beuscart, Coavoux et Maillard (2019) ont mis en place un appareillage méthodologique conséquent s'appuyant sur l'analyse, entre le $1^{\mathrm{er}}$ avril et le 31 août 2014 , de l'ensemble des consommations musicales d'un panel de près de 4000 usagers français d'une plateforme sélectionnés de manière aléatoire (pour un corpus final de 8,8 millions d'écoutes dites "qualifiées »). Cette étude quantitative devait ainsi leur permettre d'interroger la «performativité des algorithmes " des plateformes d'écoute de musique en streaming afin de nuancer la figure d'un " auditeur calculé et pris en charge [par ceux-ci], en opposition à la figure de l'amateur explorant les œuvres » (p. 26). L'objectif était donc de démontrer que, malgré les discours apocalyptiques ou laudatifs sur le supposé " pouvoir des algorithmes » (Cardon, 2018), les choix musicaux des usagers de Spotify et autres Deezer demeuraient pour une large part " autonomes », c'est-à-dire qu'ils n'auraient pas été guidés par un supplétif technique, que les auteurs qualifient d' "écoute guidée » (Beuscart, Coavoux et Maillard, 2019). Les critères permettant d'aboutir à ce 


\section{Christophe MAGIS et Lucien PERTICOZ}

résultat peuvent difficilement être discutés en détail dans le cadre du présent état de l'art, mais il en ressort toutefois qu'ils sont très largement inspirés des travaux des années 2000 autour de la diversité culturelle (cf. infra). Les auteurs concluent ainsi leur étude :

Par rapport à l'inquiétude quant à l'émergence d'un auditeur passif et hétéronome, nous montrons que, dans les usages réels, les écoutes autonomes dominent : elles représentent les trois quarts des écoutes. Autrement dit, si les plateformes de streaming dessinent en effet la figure d'un amateur complètement pris en charge par les machines, cette figure est loin d'être habitée par les utilisateurs réels, qui choisissent la majorité de leurs écoutes, et usent préférentiellement de dispositifs autonomes d'exploration tels que les moteurs de recherche et les discographies d'artistes (p. 42-43).

$\mathrm{Si}$ ces premières conclusions ne sont en soi pas si surprenantes et rejoignent les propositions de Maisonneuve (2012, 2019) sur le fait que toute innovation sociotechnique s'inscrit effectivement dans un univers de pratiques qui lui préexiste, elles nous semblent toutefois passer en partie à côté des enjeux au cœur de l'adoption massive des plateformes d'écoute en streaming par les auditeurs. Tout d'abord, l'ambition de l'appareil méthodologique mobilisé nous semble répondre à une problématique finalement assez triviale. En effet, les recherches portant sur la capacité de l'individu à faire preuve de libre arbitre face aux suggestions des algorithmes de recommandation ne sont pas sans rappeler les travaux de la sociologie nord-américaine des communications de masse, au mitan du XXème siècle, qui interrogeaient la plus ou moins grande propension des publics à adhérer aux messages diffusés par les médias de masse. À cet égard, le décalage observé entre la complexité des méthodologies déployées et des ambitions de recherche finalement assez convenues avait inspiré à Edgar Morin (1975), dans L'esprit du temps, une réflexion - un peu abrupte - qui nous semble être encore d'actualité :

Le meilleur de la sociologie américaine en matière de communication de masse s'est efforcé de réintégrer le spectateur ou le lecteur dans les groupes sociaux auxquels il appartient, mais au terme de cet effort, nous 
La musique comme analyseur : mutations de la filière musicale et mutation de la recherche sur la musique

arrivons à un point de départ : le public n'est pas une cire molle sur laquelle s'impriment les messages des communications de masse, il y a le tissu complexe des relations sociales qui interfèrent dans le rapport émetteurs-récepteurs. II n'est pas immodeste de dire qu'on s'en serait douté (p. 261).

Si nous voilà revenus au " point de départ », il convient alors peut-être de s'interroger sur la direction et les orientations prises par les pratiques des auditeurs dès qu'elles impliquent l'usage de la "marchandise musicale numérique " au sens où l'entend Morris (2015a). Et c'est précisément en ce sens que l'étude menée par Beuscart, Coavoux et Maillard (2019), pour intéressante qu'elle soit - ne serait-ce que par la taille de l'échantillon mobilisé -, nous semble manquer le coche dans la manière dont elle problématise son objet. En effet, les auteurs évoquent "l'inquiétude quant à l'émergence d'un auditeur passif et hétéronome " alors même que toute consommation culturelle nécessite une pleine activité des publics et que celleci est d'une certaine manière " attendue » par les acteurs de l'industrie. En effet, comme le rappelle Nixon :

Les membres du public ne travaillent pas seulement lorsqu'ils consomment des publicités, lorsqu'ils produisent l'idéologie de la consommation qui génère une demande pour des marchandises, mais, de façon plus exacte, ils travaillent lors de toute consommation culturelle (Nixon, 2014, cité dans Matthews, 2015, p. 65).

Ce travail de consommation culturelle apparaît encore plus essentiel quand ce qui est marchandisé est l'activité même d'écoute, de choix, voire de prescription des auditeurs qui utilisent les plateformes de streaming musical, c'est-à-dire la pratique en tant que telle (et non plus un contenu vendu sur un support édité). Cette activité est d'autant plus essentielle qu'elle permet d'enrichir en continu les algorithmes de recommandation, tels que The Echo Nest, détenu par Spotify depuis 2014. Micheau, Després-Lonnet et Cotte (2017) soulignent ainsi que les pratiques de consommations musicales génèrent toute une série d' " inscriptions documentaires ». À cet égard, la playlist représente, selon les auteurs, l'une des concrétisations les plus exemplaires de la documentarisation (et donc de la marchandisation) des pratiques d'écoute musicale en régime numérique. L'enjeu pour ces plateformes 


\section{Christophe MAGIS et Lucien PERTICOZ}

ne se situe donc pas tant dans la manière (" hétéronome " ou " autonome ») de choisir d'écouter tel ou tel morceau que dans le fait d'effectivement poser des choix sur tel ou tel morceau, dans tel ou tel lieu, à tel ou tel moment, avec telle ou telle personne. Ce qui importe en définitive, c'est que l'auditeur écoute de la musique sur ledit dispositif. Celui-ci ne sert donc pas tant à rendre les auditeurs plus " passifs ", mais bien plutôt à encadrer une activité qui est elle-même encouragée, ce cadrage favorisant ensuite la valorisation marchande de leurs pratiques. Reprenant les propositions de Le Guern (2016) à ce sujet, les auteurs suggèrent que ces plateformes de streaming entendent contribuer à la constitution d'une "politique des affects et des goûts » en régime numérique et " dont l'objectif final est bien sûr de non seulement capter de l'audience, mais aussi des usagers qui, par leurs activités sur la plateforme, vont nourrir le système de recommandation » (Micheau, DesprésLonnet et Cotte, 2017, p. 45). Ces propositions rejoignent particulièrement celles formulées par Stéphan-Éloïse Gras (2014, 2015) qui, dans le cadre d'une étude fine de l'algorithme de recommandation The Echo Nest croisant une archéologie de l'écoute, une étude des politiques de l'archive et de l'algorithme ainsi qu'une analyse sémiopragmatique de son interface, a montré en quoi cette " machine du goût " contribue à façonner les expériences musicales en régime numérique. Elle estime ainsi que l'enjeu industriel pour les acteurs économiques qui les détiennent (ici Spotify) ne se situerait pas tant dans leur capacité supposée à orienter les auditeurs vers tel ou tel titre, mais bien davantage à construire ce qu'elle nomme une "discipline de l'écoutable " qui "ne suppose pas une contrainte directive sur ce qui est écouté, mais plutôt une grammatisation de la gestuelle de l'écoute " (Gras, 2015, p. 81), c'est-à-dire une manière de normer et de contrôler la pratique d'écoute afin de faciliter sa captation industrielle.

En effet, à partir du moment où c'est l'acte d'écoute qui devient marchandise, il convient dans une certaine mesure d'être capable de l'industrialiser, ce qui, du point de vue des acteurs économiques, constitue la principale "promesse » du numérique. Manuel Dupuy-Salle relève à cet égard que si, en 2017, une plateforme telle que Deezer mettait en avant sa capacité à articuler « expérience d'écoute et [...] découverte musicale personnalisée et augmentée » (Dupuy-Salle, 2019, p. 89), c'est tout d'abord pour se distinguer de la concurrence, 
La musique comme analyseur : mutations de la filière musicale et mutation de la recherche sur la musique

alors même qu'il n'existe pas de réelle politique commerciale d'exclusivité sur le marché du streaming musical, rendant impossible une différenciation de l'offre par le catalogue de titres proposés. Surtout, l'auteur souligne plus loin que ces services de prescription "sont également utilisés pour mettre en avant des contenus faisant l'objet de partenariats avec des acteurs de la musique [...] ou des médias » (p. 91). C'est donc bien la pratique d'écoute en tant que telle qui rend possible une transaction marchande, ce qui nécessite effectivement qu'elle soit « produite » de manière un tant soit peu rationalisée.

Ce postulat n'est pas sans conséquence sur la manière dont il convient, dès lors, d'interroger les évolutions des pratiques d'écoute musicale en régime numérique ainsi que les modalités de leur exploitation marchande. Comme le souligne Philippe Bouquillion (2020), cela nécessite en premier lieu de ne pas uniquement se focaliser sur ce qu'il nomme la « dimension idéelle » de la recommandation - qui sous-tend les approches relevant de l'économie de l'attention - , mais de réinscrire ces recherches dans le cadre d'une économie de l'incertitude ; « les pratiques culturelles sont alors vues comme étant radicalement imprévisibles", alors même que "les filières des industries culturelles sont organisées pour mettre sur le marché plus de contenus que les consommateurs ne pourront jamais en absorber » (p. 24-25). L'auteur en conclut que « la dimension stratégique de la recommandation peut [alors] être mise en tension avec sa fonction affichée, celle de l'appariement de l'offre et de la demande " (p. 25). À notre sens, ceci implique également d'interroger la manière dont les pratiques de consommation de contenus musicaux (et culturels au sens large) sont produites par des acteurs économiques dont les intérêts ne convergent pas nécessairement, un chantier qui relèverait dès lors d'une économie politique des pratiques culturelles (Perticoz, 2012b). Celui-ci pourrait notamment s'inspirer des recherches menées sur les plateformes de crowdfunding et de crowdsourcing (Rouzé, Matthews et Vachet, 2014 ; Rouzé et Matthews, 2018), sur les usages de Facebook par les artistes musicaux (Creton, 2018) ou, plus récemment, sur les promesses de la technologie blockchain (Magaudda, 2019), qui, chacune à leur manière, montrent comment, au-delà des tensions qui traversent les rapports entre les différents acteurs économiques qui les exploitent, les stratégies marchandes qui orientent les usages de ces innovations « nous 


\section{Christophe MAGIS et Lucien PERTICOZ}

[amènent] un pas plus loin sur la voie de la monétisation des pratiques et des goûts musicaux » (Magaudda, 2019, p. 865).

\section{Mutations numériques et transformation de la réflexion sur les « contenus » musicaux}

Les études de communication, et notamment en leur sein les analyses des industries culturelles, sont traversées de tensions entre, d'un côté, des approches se concentrant tantôt sur la socioéconomie des structures de production et, de l'autre, des travaux qui s'attachent à définir les modalités de construction du sens. Le corpus des recherches sur les transformations de la filière musicale et, d'une manière plus générale, le corpus des popular music studies ne font pas exception : la nécessité de faire tenir ensemble l'analyse des conditions de production ou de distribution, la description de la nature de l'expérience de l'écoute ou les différents statuts du texte musical a résulté en un ensemble de travaux issus "de la musicologie, de la sociologie et des études culturelles et médiatiques, qui ne s'accordent pas sur ce qui constitue le cœur principal de l'analyse " (Shuker, 2008, p. 2). De cette tension a résulté ce qu'Antoine Hennion (1998) appelle une «distribution fâcheuse ", qui voit généralement les diverses approches se répartir selon les différentes esthétiques: "Aux musiques sérieuses, savantes, autonomes les subtilités de l'analyse musicale, aux musiques populaires de tous ordres les analyses culturelles, sociales, ethnologiques, rituelles, économiques... » (p. 13).

De fait, dans le cadre général des travaux sur les industries culturelles, les tentatives véritables d'articulation de l'analyse musicale ou esthétique à l'analyse socioéconomique des mutations de la filière musicale ont été bien rares. Pourtant, on imagine combien une telle approche, qui vise à appréhender les industries de la culture, de l'information et de la communication en prêtant attention au "rôle qu'elles jouent dans la vie culturelle, sociale et politique d'un pays " (Tremblay, 2007, p. 207), pourrait tirer d'une évaluation précise des " contenus». Les mutations numériques de la musique ont notamment vu un certain nombre de travaux visant à s'approcher de cette évaluation. Nous les présentons dans cette dernière partie, en essayant de mettre en avant le 
La musique comme analyseur : mutations de la filière musicale et mutation de la recherche sur la musique

mouvement possible vers une articulation plus poussée de l'analyse socioéconomique et de l'analyse musicale.

\subsection{Capitalisme, diversité et expression démocratique}

La crise du disque a, comme on peut s'y attendre, alimenté un certain nombre de questionnements institutionnels. II s'est alors opéré une récupération d'une thématique ancienne, mais dont l'usage s'est quelque peu transformé : celle de la diversité.

Comme le note Tristan Mattelart (2011), «la notion de "diversité culturelle" a, pour elle, l'attrait de l'apparente évidence. Comment pourrait-on être contre le pluralisme qu'elle implique ? " (p. 29.) Or l'auteur rappelle toutefois combien la polysémie du terme pose problème, lui permettant d'être tour à tour endossé par des discours accompagnant des intérêts contradictoires, au sein de programmes politiques divergents ou de projets théoriques antagonistes, et souvent non critiques. II n'est alors pas inutile de s'intéresser à l'utilisation continue de cette notion dans les travaux sur les mutations numériques de la musique.

Pour rappel, c'est au départ autour des risques que les phénomènes de globalisation faisaient courir à l'autonomie culturelle des pays du Tiers-Monde et des anciennes colonies que la thématique de la diversité s'est développée à partir de la fin des années 1960. Pour permettre à ces pays le développement d'une offre culturelle propre dans la concurrence internationale de la culture commerciale, un certain nombre de travaux ont milité auprès d'institutions nationales et supranationales (par exemple, de I'UNESCO) pour l'élaboration de politiques culturelles autonomes ou favorisant cette autonomie. C'est le début d'une formalisation du concept de "diversité culturelle», souvent pensé comme corollaire de la diversité biologique :

En 1972, la Conférence des Nations unies sur l'environnement et le développement de Stockholm associe le thème de la défense de la biodiversité à celui de la diversité culturelle, réalité toutes deux menacées par les logiques prédatrices et inégalitaires du modèle de croissance occidental mû par la consommation excessive des ressources naturelles comme des biens matériels. Vingt ans plus tard, le Sommet de la Terre de Rio 


\section{Christophe MAGIS et Lucien PERTICOZ}

conjugue la diversité culturelle avec le concept de "développement durable » [...]. C'est d'ailleurs par l'entremise du développement durable que des organisations comme la Banque mondiale incorporent la culture dans leurs préoccupations à l'aube du troisième millénaire. En plaidant pour l'équilibre des « écosystèmes culturels ", l'Unesco introduit le thème de la diversité dans sa philosophie et ses plans d'action dans les années 1990. (A. Mattelart, 2007, p. 97).

Rapidement reprise par de nombreux travaux, la thématique a permis d'appuyer l'importance de la sauvegarde de la souveraineté nationale en matière de production médiatique (Mattelart, Delcourt et Mattelart, 1983; Nordenstreng et Schiller, 1979), ou de l'« autonomie culturelle " (Hamelink, 1983), et ce, notamment à propos d'industries comme le cinéma, économie de prototypes aux coûts de production élevés. À partir de la fin des années 1970, la diversité a également été la catégorie par laquelle ces travaux ont été critiqués par des théoriciens du postmodernisme avec un changement de perspective : il se répand l'idée « que l'un des principaux lieux de production de la diversité culturelle est la consommation. Ce déplacement est largement lié au développement des recherches sur la réception de la télévision » (T. Mattelart, 2011, p. 26).

Petit à petit, la diversité devient alors une catégorie économique pour penser les mutations internationales à l'œuvre dans les industries médiatiques. Dans les années 1990, les négociations du General Agreement for Tariffs and Trade (GATT) $)^{13}$, mais surtout de l'Organisation mondiale du commerce (OMC) et de I'UNESCO s'emparent alors de la thématique. Elle devient un élément de réflexion et d'évaluation des politiques culturelles. C'est ainsi qu'en France, par exemple, le Département des études, de la prospective et des statistiques (DEPS) du ministère français de la Culture publie régulièrement, depuis le début des années 2000 , des études sur l'évolution de la diversité dans les différentes filières des industries culturelles. On sait combien l'histoire des

13 Traité de libre-échange signé en 1947 par 23 pays dans le but d'harmoniser leurs politiques douanières. Jusqu'à une centaine de pays ont rejoint cet accord et ses diverses négociations. Son domaine de compétence a été élargi avec la création de l'OMC, qui l'a remplacé en 1995. 
La musique comme analyseur : mutations de la filière musicale et mutation de la recherche sur la musique

politiques culturelles en France est caractérisée par la volonté de préserver un rayonnement culturel français et francophone, notamment à partir de la création du ministère de la Culture confié à André Malraux en 1959. Une expression comme celle d' " exception culturelle française ", par laquelle ont été mis en place des systèmes d'intervention de l'État dans le soutien de la culture en France (subventions, quotas...), en est une des manifestations les plus connues. Ayant vu son domaine d'intervention considérablement élargi depuis les années 1980 et les " années Lang " avec la formule " économie et culture : même combat » lancée par l'ancien ministre de la Culture devant l'assemblée dès 1981, dans une période de mise à l'honneur de la culture comme lieu d'investissement économique rentable pour un pays commençant son déclin industriel, cet interventionnisme s'est accompagné de la production d'indicateurs à même d'aider à justifier les propositions politiques en direction de la culture, surtout dans un contexte économique qui leur est généralement hostile ${ }^{14}$. Ces indicateurs sont d'ailleurs toujours au centre de combats politiques à propos de la culture où les dimensions économiques et symboliques s'imbriquent. Nous reviendrons ciaprès sur la question de ces indicateurs, mais il est nécessaire de remarquer avant tout, et à la suite de Bouquillion et Combès (2011), combien, à partir du début des années 2000 et dans la suite des mouvements altermondialistes du tournant du XXIème siècle, la thématique de la diversité s'articule à celle de la démocratie. La diversité est alors en effet présentée " comme un gage de l'expression démocratique, du bon fonctionnement de l'espace public politique et de la participation citoyenne, en particulier du fait de l'une de ses facettes, le pluralisme » (Bouquillion et Combès, 2011, p. 9). Si les ancrages normatifs des études de la diversité sont bien

14 On consultera pour s'en persuader l'analyse par Mattelart, Delcourt et Mattelart (1983, p. 26-31) de la réception du discours de Jack Lang à la convention de I'UNESCO à Mexico sur les politiques culturelles de 1982 dans les médias, notamment français: "Oblitérant la conjonction entre économie et culture, la polémique répercutée par les médias français se focalise sur "l'impérialisme culturel américain" [...] et vire à la bataille des jugements de valeur et des procès d'intention. Le ministre français se voit accusé d'avoir déclenché "la guerre des bérets, des bourrées, des binious" contre Dashiel Hammett, Chester Himes, William Irush, Orson Welles, Meredith Monk, Richard Forman, Pollock, Warhol, Cunningham, etc. "La plus nulle des revues de Broadway surclassera toujours l'affligeant spectacle des danses folkloriques en sabots", écrit l'un des plus féroces critiques. " (Mattelart, Delcourt et Mattelart, 1983, p. 29.) 


\section{Christophe MAGIS et Lucien PERTICOZ}

rarement explicités, on peut en effet constater, en s'insérant dans une réflexion critique, que, d'une manière générale, dans les travaux, le problème de la diversité se réduit à un questionnement de l'ordre suivant: tandis que le mode de production capitaliste étend le règne de la marchandise sur la culture, les conditions d'une représentativité multiple des différentes propositions et discours culturels sont-elles garanties?

On sait que le capitalisme du XX ${ }^{\text {ème }}$ siècle, contrairement au catéchisme habituellement colporté par les défenseurs du libre marché, ne tend pas vers une saine concurrence entre les différents offreurs qui engendrerait une meilleure innovation et une qualité toujours supérieure pour des prix toujours les plus bas possibles: il tend au contraire à la constitution de monopoles (Baran et Sweezy, 1968). Au sein des différents secteurs productifs, les industries culturelles et médiatiques sont connues pour être paradigmatiques de ce mouvement vers la constitution de monopoles, entre autres en raison de certaines de leurs spécificités socioéconomiques, qui avantagent toujours les acteurs les plus concentrés (Bouquillion, 2008 ; Doyle, 2002). Une des conséquences en étant la multiplication de situations de non-concurrence (ou de concurrence "aménagée », c'est-à-dire les oligopoles), une question se pose quant au risque d'érosion de la pluralité des discours ou imaginaires disponibles.

La crise, et les mutations numériques apportent un déplacement progressif de cette question, comme on le comprend à lire l'étude sur l'évolution de la diversité dans la filière musicale publiée par le DEPS en 2011 :

Sans que l'on cherche ici à identifier l'ampleur ni même l'existence d'une causalité entre les deux phénomènes, la crise des ventes de musique enregistrée s'inscrit en parallèle d'un processus avancé de numérisation de la filière. En matière de diversité, l'industrie de la musique enregistrée offre donc un éclairage spécifique : comment évolue la diversité culturelle dans une industrie en crise ? Comment les ventes en ligne influent-elles sur la diversité ? Le poids conjugué très important de la grande distribution alimentaire et des grandes surfaces spécialisées dans les produits culturels dans les ventes de 
La musique comme analyseur : mutations de la filière musicale et mutation de la recherche sur la musique

musique enregistrée a-t-il des conséquences en matière de diversité ? (Bourreau, Moreau et Senellart, 2011, p. 1)

D'un côté, la crise de la vente des supports, avec pour conséquence la révocation d'un certain nombre de contrats d'artistes au sein des maisons d'édition, fait courir le risque d'une production moins variée, se recentrant sur les titres et les interprètes stars. De l'autre, la numérisation a engendré de nouveaux modes d'écoutes (via le téléchargement puis les sites de diffusion en streaming) dans lesquels la recommandation devient prégnante. II s'agit alors d'étudier la diversité consommée ${ }^{15}$. Dans tous les cas, c'est toujours cette idée abstraite de pluralité des représentations qui est au centre des études. On peut alors s'interroger sur sa légitimité scientifique. Suffirait-il que la production et la consommation soient "diversifiées" pour estimer que le problème de la représentation démocratique est résolu ?

Par ailleurs, il convient d'affiner les indicateurs par lesquels ces études sont menées, car, « en conséquence de [sa] dimension polysémique, il semble difficile de mesurer la diversité " (Bouquillion et Combès, 2011, p. 10), notamment pour l'économie. De toute évidence, on ne peut l'identifier à la seule variété (ou multiplicité - c'est-à-dire le nombre de titres différents) produite, surtout dans un secteur caractérisé par l'abondance de l'offre et la surproduction. En s'appuyant sur les indicateurs issus des sciences biologiques (Rao, 1982), les économistes de la culture se sont entendus, à la suite de Stirling (1998), sur trois critères: variété (quantité de titres produits), équilibre (homogénéité dans les proportions de vente des différents titres) et disparité (degré de différence entre les différents titres produits). Sauf que si « [l']évaluation quantitative de la variété et de l'équilibre est simple : la variété est un simple entier positif et l'équilibre est proche de la variance ॥, il n'en est pas de même pour le troisième

\footnotetext{
15 À ce propos, l'hypothèse de la "Longue traîne » (Long Tail) formulée par Chris Anderson en 2004 est régulièrement au centre des débats. Partant du postulat que, quand le coût d'opportunité est élevé (comme dans le cas de la distribution physique de la musique), seuls les produits qui concentrent le maximum des achats est proposé à la vente, Anderson pose que si les coûts de stockage et de distribution sont faibles (comme dans le cas de la distribution en ligne) alors on peut virtuellement proposer des produits plus diversifiés: le marché cumulé des titres rarement demandés est plus important que celui des produits qui concentrent le maximum d'achat.
} 


\section{Christophe MAGIS et Lucien PERTICOZ}

indicateur : " la disparité se révèle bien plus difficile à évaluer » (Moreau et Peltier, 2004, p. 126).

Après quelques tentatives de mise au point méthodologique (dont certaines ont vu les travaux faire tout simplement l'impasse sur la disparité), les chercheurs se sont globalement entendus sur un certain nombre de variables quantitatives pour mesurer chaque indicateur. Les études réalisées pour le DEPS par Marc Bourreau, François Moreau et Pierre Senellart sur la " diversité culturelle dans la musique enregistrée en France " (2011, p. 15) et par Olivier Donnat sur l'« évolution de la diversité consommée sur le marché de la musique enregistrée, 2007-2016 » (2018, p. 27) donnent, pour mesurer la disparité, pas moins de onze variables dont certaines sont intéressantes, notamment les " taux de renouvellement dans les tops " et les " taux de similitudes entre les tops et les circuits", à la fois pour les titres et pour les artistes, ou le «nombre d'éditeurs actifs ». Mais l'utilisation de tels paramètres de mesure pour évaluer le "degré de différence " entre les titres produits ou consommés tend à produire une forme de tautologie : en lieu et place d'une mesure de cette différence, on a une mesure de la concentration, répercutée sur la chaîne de production depuis les grosses entreprises médiatiques jusqu'aux éditeurs, aux artistes, puis aux titres mêmes. Pour le dire autrement, l'analyse économique pose quelque peu ses postulats comme méthodologie d'analyse.

Nous voyons donc combien les mutations numériques de la musique sur les deux dernières décennies sont l'occasion d'une reprise de la thématique de la diversité qui n'est pas sans poser encore un certain nombre de limites que de prochains travaux auront à résoudre. Comment ancrer normativement la nécessité abstraite de la diversité autrement que par une critique des idéologies? Et comment permettre à une telle analyse de se doter d'indicateurs à la hauteur de cette critique?

\subsection{De la diversité à la « découvrabilité » : vers une étude de la recommandation?}

Parmi les thématiques qui ont émergé ces dernières années autour des enjeux des révolutions numériques, celle de l'« économie de l'attention" (Boullier, 2009; Citton, 2014; 
La musique comme analyseur : mutations de la filière musicale et mutation de la recherche sur la musique

Lanham, 2006) est au nombre des plus ambitieuses. Sa proposition pourrait être ainsi résumée: la place de plus en plus prégnante prise par les technologies de l'information et de la communication dans notre quotidien, associée à certaines spécificités économiques des biens informationnels (caractère de bien public, de bien d'expérience, aspect négligeable du coût de leur reproduction par rapport à celui de leur production, par exemple) conduirait vers un déplacement de la rareté (catégorie fondamentale de la réflexion économique) depuis la production vers la réception. Tandis que l'économie a traditionnellement toujours supposé une rareté des ressources conduisant à la production des biens offerts, justifiant un prix de vente pour départager les demandeurs, l'époque actuelle serait au contraire celle d'un renversement: les biens sont disponibles de manière surabondante et généralement gratuitement ; l'enjeu économique principal résiderait alors dans l'orientation du consommateur à travers une telle offre pléthorique (Citton, 2014). La discussion de telles propositions a, bien évidemment, été au cœur d'une réorientation des problématiques des industries culturelles et médiatiques, en général, et de la filière musicale en particulier.

L'intérêt porté à la question des évolutions des environnements sonores n'est pas nouveau. Dans le sillage de travaux initiés dès les années 1960 et du « World Soundscape Project " créé en 1973, le compositeur canadien Raymond Murray Schafer s'attache dès les années 1970 à définir en musicien les marqueurs sonores caractéristiques de l'identité acoustique des différents lieux ${ }^{16}$. Plusieurs autres propositions pour une "écologie sonore " lui ont emboîté le pas (Truax, $1978,1985)$, s'appuyant sur une conception du monde comme une sorte de gigantesque composition musicale proche des travaux du compositeur américain John Cage. La constitution et la commercialisation de banques sonores, puis la numérisation de la musique et l'explosion conséquente de sa diffusion dans des situations d'écoute toujours plus vastes et renouvelées n'ont que faiblement accéléré l'intérêt porté à ces thématiques, notamment au-delà des seuls enjeux esthétiques. L'ouvrage de Joseph Lanza (2004) sur la "musique d'ascenseur » et la thèse de Vincent Rouzé (2004) sur les musiques diffusées dans

16 Ses différents travaux titrés The New Soundscape ont été rassemblés dans l'ouvrage The Soundscape en 1993 (traduction française en 2010). 


\section{Christophe MAGIS et Lucien PERTICOZ}

les lieux publics étudient les multiples facettes de la mise en musique du quotidien.

La "Muzak », comme on l'appelle parfois par antonomase du nom de la société américaine pionnière dans le domaine de la musique d'ambiance, n'a jamais eu la faveur des mélomanes. Pourtant, on pourrait considérer à bien des égards, avec Lanza (2004), qu'elle est « esthétiquement supérieure à toute autre forme musicale : elle émet la musique comme le $X X^{\text {ème }}$ siècle est équipé pour la recevoir ${ }^{17} »$ (p. 5). Elle n'a guère eu non plus la faveur des chercheurs à l'exception d'un courant américain du marketing d'inspiration behavioriste qui, à partir des années 1980, a lancé la revue Marketing Through Music, dans laquelle ont été publiées plusieurs études sur les réactions des consommateurs selon leur exposition à divers stimuli musicaux en situation d'achat (Bruner, 1990, pour un panorama). Pourtant, les musiques d'ambiance, ainsi que plusieurs autres musiques produites "à la demande " (Faulkner, 1983), comme la musique de film ou de publicité, selon un certain nombre de contraintes d'ordre stylistique ou esthétique, sont un objet de choix pour l'étude de la mise en rapport entre les différentes contraintes techniques, commerciales ou communicationnelles et des contenus musicaux (Scherzinger, 2005). L'étude de la musique de publicité proposée par Christophe Magis (2015) révèle ainsi les différentes stratégies de rationalisation des services spécialisés de conseil en communication sonore pour la mise en relation entre des particularités musicales et des situations de communication. Or il a fallu attendre l'essor des services de streaming, et leur catégorisation précise des rapports entre les titres musicaux et les goûts des amateurs dans le cas d'algorithmes complexes, pour que les chercheurs s'intéressent plus précisément au fonctionnement de telles catégorisations. Et pour cause, le succès du streaming musical comme mode d'accès à la musique (figure 3), parfois à l'intermédiaire de plateformes généralistes (YouTube), mais surtout de plateformes spécialisées (Deezer, Spotify) donnant accès à un catalogue tendant vers l'exhaustivité, semble être une simple

\footnotetext{
17 Traduction libre de : « Muzak and mood music are, in many respects, aesthetically superior to all other musical forms: they emit music the way the twentieth century is equipped to receive it. »
} 
La musique comme analyseur : mutations de la filière musicale et mutation de la recherche sur la musique

confirmation de l'hypothèse principale de l'économie de l'attention dans le secteur musical.

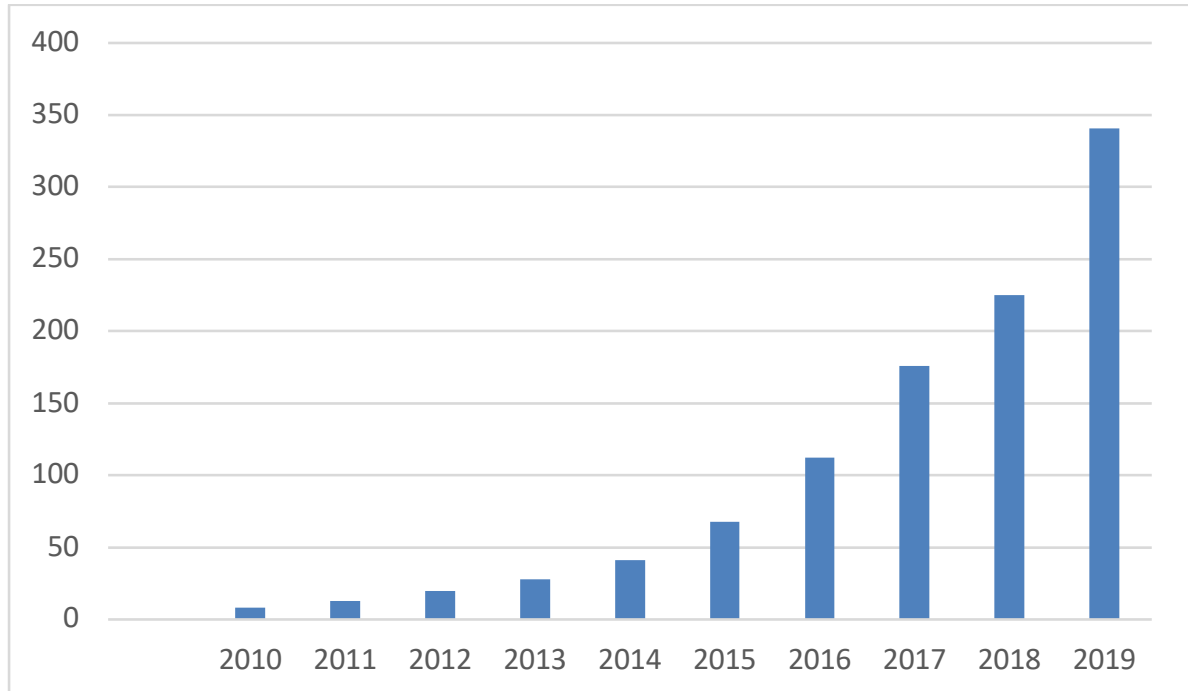

Figure 3. Évolution du nombre d'abonnés payants à un service de streaming musical dans le monde, entre 2010 et 2019 (en millions d'abonnés) (source : IFPI - Global Music Report - The Industry in 2019)

Dans le sillage des études sur les sites de e-commerce, les plateformes ont donc également fait l'objet de recherches quant à leurs systèmes de recommandation, et ce, dans un premier temps par rapport à la question de leur impact sur la " diversité » (Celma et Cano, 2008). Plus récemment, c'est le fonctionnement des algorithmes de découverte musicale des plateformes qui a été au cœur de l'attention et plusieurs travaux s'attardent à en décrire les principes. Ainsi, Robert Prey (2018) décortique, pour les comparer, les algorithmes de recommandation du service de webradio Pandora (qui intègre le système de recommandation musicale créé par le Music Genome Project) et de Spotify. Le premier se distingue du fonctionnement habituel des radios comme des sites de vente, qu'ils soient physiques ou en ligne: il ne rassemble les chansons ni par genre, ni par association statistique de type " les auditeurs qui ont aimé tel titre ont aussi aimé tel autre » ni par système de notation. Au contraire, Pandora "classe la musique selon des caractères musicaux ou "gènes" » qui peuvent comprendre "le genre du chanteur, le tempo du refrain, le niveau de distorsion de la guitare électrique, le type 


\section{Christophe MAGIS et Lucien PERTICOZ}

de chœurs, etc. ${ }^{18}$ " (Prey, 2018, p. 1089). Ces "gènes » musicaux varient selon les genres musicaux. Aussi, leur production pour chaque titre nécessite un important travail humain de classification. De leur côté, les auditeurs sont également caractérisés par leur réceptivité à ces différents gènes. Pour sa part, le système de recommandation de Spotify combine deux logiques: "celle dite collaborative filtering et, d'autre part, celle dite content-based filtering" (Bouquillion, 2020, p. 22). II articule à la fois une analyse sémantique de tous les commentaires concernant les titres à une analyse de caractéristiques sonores, analysables automatiquement à partir du fichier son au moyen de l'outil The Echo Nest qui " transforme chaque conversation à propos de musique et la musique elle-même en données quantifiables ${ }^{19}$ " (Prey, 2018, p. 1091).

Dans le cas de Pandora comme de Spotify, l'enjeu fondamental de la recommandation est d'établir parallèlement une cartographie des auditeurs susceptible de se conformer aux segmentations qui régissent les marchés publicitaires, et ce, y compris dans le cas de services premium payants (Morris, 2015b ; Prey, 2018). On constate alors combien la capacité d'un système de recommandation à retenir un auditeur " captif » est progressivement devenue la variable principale pour juger de sa pertinence (Seaver, 2018). Par ailleurs, même lorsque les utilisateurs n'y ont pas ou peu recours, la recommandation personnalisée est importante dans leur appréciation de la qualité globale des services et, partant, elle constitue un argument de promotion des offres (Farchy, Méadel et Anciaux, 2017). À ce propos, au milieu de ces évolutions des systèmes de recommandation, plusieurs travaux québécois, souvent commandités par diverses institutions francophones, interrogent une nouvelle catégorie fondamentale dans la distinction des offres de streaming musical: celle de " découvrabilité $»^{20}$. II s'agit notamment d'interroger l'incidence

18 Traduction libre de: "Pandora organizes music by musical traits, or "genes". Genes could include, for example, the gender of the lead vocalist, the tempo of the chorus, the level of distortion on the electric guitar, the type of background vocals, and many more. "

19 Traduction libre de: "Essentially, The Echo Nest attempts to turn both conversations about music, and music itself, into quantifiable data. "

20 La catégorie a ainsi fait l'objet d'une conférence internationale à l'Université du Québec à Montréal en octobre 2019, dans le cadre de l'Organisation internationale de la Francophonie. 
La musique comme analyseur : mutations de la filière musicale et mutation de la recherche sur la musique

des systèmes sociotechniques associés à la recommandation musicale en ligne sur la mise en avant ou l'invisibilisation éventuelle de certaines œuvres du répertoire francophone (Maisonneuve, 2019 ; Roberge, Jamet et Rousseau, 2019).

\subsection{Pour une articulation de l'analyse musicale à l'analyse socioéconomique}

Les analyses du fonctionnement algorithmique des systèmes de recommandation des plateformes de streaming musical tendent, bien que de manière indirecte, à révéler la faiblesse méthodologique fondamentale des études habituelles de la diversité dans la musique. En effet, on s'aperçoit que, pour penser les rapports entre les différents titres, les plateformes elles-mêmes utilisent des indicateurs bien plus précis que ceux des chercheurs qui s'appuient sur la méthodologie de Sterling! On peut ainsi espérer que la nécessaire critique de la réification inhérente au principe de rationalisation algorithmique encouragera de prochaines recherches se proposant d'intégrer des formes d'analyse "content-based" à la critique socioéconomique des mutations de l'industrie musicale dans le capitalisme. Si ce champ d'investigation reste encore largement à ouvrir, il pourra s'appuyer sur les quelques propositions d'ores et déjà existantes d'articulation des analyses socioéconomique et musicale.

Les écrits d'Adorno "sur la musique populaire " (Adorno, 2010), "sur le jazz » (Adorno, 1994) ou sur la " musique de cinéma » (Adorno et Eisler, 1969) sont souvent présentées comme un repoussoir dans les popular music studies. On n'en retient généralement que le caractère polémique et péremptoire de certains passages et on critique en retour le manque de connaissance du philosophe quant aux diverses esthétiques des musiques populaires, de même que son jugement " élitiste » à l'aune de critères hérités du répertoire savant, mais on tend souvent à oublier le cadre théorique général qui sous-tend ses propositions: la critique des matérialisations culturelles des évolutions du capitalisme libéral vers un capitalisme caractérisé par les monopoles, produisant une civilisation qui "confère à tout un air de ressemblance " (Horkheimer et Adorno, 1974, p. 129). Replacés dans ce cadre, ces travaux sont une proposition de départ d'« analyse de marchandises musicales » (Adorno, 1982, p. 43-57), d'ailleurs 


\section{Christophe MAGIS et Lucien PERTICOZ}

souvent indépendamment de leur caractère savant ou populaire, comme autant de totalités sociales et culturelles par lesquelles cette civilisation spécifique du capitalisme peut être étudiée. À cet égard, les deux catégories essentielles par lesquelles Adorno se propose de caractériser le fonctionnement des œuvres musicales industrialisées sont la standardisation et la pseudo-individualisation. La première révèle combien chaque titre s'attache à suivre le schéma formel des chansons à succès (même construction dans l'alternance couplets/refrains, mêmes structures harmoniques, métriques semblables...), duquel les principes socioéconomiques qui dirigent la production ne permettent guère de s'éloigner, par aversion pour le risque. La seconde a pour but de " camoufler le prédigéré ", c'est-à-dire de maquiller ce caractère standardisé des titres, au moyen d'effets musicaux ou paramusicaux, par exemple les types d'arrangements et d'orchestration, la construction de discours autour de la personnalité des interprètes, etc. (Adorno, 2010.)

Le musicologue Richard Middleton a été l'un des premiers à s'appuyer sur de telles catégories pour une analyse systématique des œuvres musicales de la culture de masse. II montre ainsi comment la production de musique populaire d'après-guerre, en continuant de fonctionner selon ces deux critères, a considérablement élargi les possibilités de «pseudoindividualisation » :

En raison de l'ubiquité de la musique populaire et l'échelle vaste de sa production, il a été possible d'établir un panel de "couleurs " musicales conventionnelles dans l'esprit collectif - " hispanique », " pastoral », « cowboy », " blues ", " hippie », " punk » etc. - et les arrangeurs ou les producteurs peuvent au besoin se servir dans la bibliothèque des procédés techniques de ces placages préétablis. De la même manière, la «spontanéité mécanisée " typique des certains orchestres de danse de la fin des années 1930 se retrouve dans l'excitation artificielle et l'extase chiquée de certaines performances rock - tout comme les solos "improvisés " sont encore communément pré-écrits. D'une certaine manière, n'importe quoi - même la dissonance " moderniste " ou le phrasé "anti-vocal» des chanteurs de punk - peut devenir un cliché de pseudo-individualisation, comme bon 
La musique comme analyseur : mutations de la filière musicale et mutation de la recherche sur la musique

nombre de publicités télévisuelles en attestent ${ }^{21}$ (Middleton, 1990, p. 50).

L'analyse de ces procédés de pseudo-individualisation peut ainsi s'étendre jusqu'au rôle des langues mêmes dans lesquelles les tubes internationaux sont chantés (Magis, 2020). Néanmoins, on peut tout à fait imaginer qu'elle puisse servir à la critique des différents types d'articulations entre des matériaux musicaux et des "styles de vie " dans le profilage social des auditeurs par les algorithmes des plateformes. À cet égard, l'étude des contraintes qui s'imposent aux producteurs de musique de publicité pour mettre en relation des contraintes commerciales et communicationnelles avec des caractéristiques musicales peut être un point de départ (Magis, 2015). Bien qu'elles ne soient pas tout à fait standardisées, mais dépendantes des appréciations différentes des producteurs, ces articulations (comme l'emploi du jazz pour donner une tonalité " classe » à un message publicitaire, par exemple), assez récurrentes, semblent souvent prêtes à faire l'objet d'une utilisation algorithmique.

De futures recherches pourront ainsi, sans se séparer des analyses sociologiques concernant les usages des publics ni des travaux d'économie politique sur les transformations des structurations du secteur, s'appuyer également sur le corpus des analyses des musiques populaires. À titre d'exemple, certains travaux canoniques ont déjà quelque peu exploré le terrain, comme la " sociologie des variétés » d'Antoine Hennion (1982), qui ne s'interdit pas la tentative d'étudier aussi le sens des paroles et de la musique des chansons tout en étudiant les modalités de leur production, les études d'Howard Becker $(1985,2003)$ sur les praticiens de musique, qui s'appuient sur des observations participantes, ou encore celles, nombreuses,

21 Traduction libre de : "Because of popular music's ubiquitousness and vast scale of production, it has been possible to establish in the collective mind a set of conventional musical "colours" - "Spanish", "pastoral", "cowboy", "blue", "hippie", "punk", and so on - and arrangers and producers can simply lift the technical devices needed for the ready-made veneers off the shelf when needed. Similarly, the 'mechanized spontaneity' typical of some late 1930s dance-bands can easily be matched by the artificial excitement and synthetic ecstasy of some rock performances - just as rehearsed 'improvised' solos are still common. In a sense, anything - even "modernist" dissonance or punk 'anti-vocal' singing - can become clichéd and pseudo-individual, as many TV commercials testify. » 


\section{Christophe MAGIS et Lucien PERTICOZ}

de Philipp Tagg ${ }^{22}$, qui s'intéresse tant aux mécanismes de production de sens dans la musique de télévision, dans les chansons populaires (à partir de la voix, notamment), ou des rapports de la musique à l'identité, depuis une perspective originale articulant sociologie et musicologie.

Certains travaux sur les musiques populaires qui s'élaborent dans le monde francophone autour de la revue Volume! nous paraissent ainsi à prendre très au sérieux pour les chercheurs qui étudient les mutations des industries culturelles, fût-ce depuis une perspective économique ou sociologique. Et nous espérons que de prochains projets pourront ainsi articuler, pour penser les mutations de la filière musicale dans une perspective critique, la socioéconomie de la production, les études de réception ou encore la sociologie des usages du numérique et la production musicale du sens, fidèles en cela à la tradition interdisciplinaire des études de communication.

\section{Références}

Adorno, T. W. (1982). Quasi una fantasia, Paris, France: Gallimard.

Adorno, T. W. (1994). Introduction à la sociologie de la musique. Genève, Suisse : Contrechamps.

Adorno, T. W. (2010). Current of Music. Éléments pour une théorie de la radio. Paris, France/Québec, Canada: MSH/Presses de l'Université Laval.

Adorno, T. W. et Eisler H. (1969). Musique de cinéma. Paris, France : L'Arche.

Attali, J. (1977). Bruits. Essai sur l'économie politique de la musique. Paris, France : Presses universitaires de France.

Baran, P. et Sweezy, P. (1968). Le capitalisme monopoliste : un essai sur la société industrielle américaine. Paris, France: Éditions Maspero.

Battisti, M. (2004). Accès à l'information et droit d'auteur : une solution équilibrée est-elle encore possible? Débat de

22 Disponibles sur son site : https://tagg.org/index.html (dernière consultation le 25 juillet 2020). 
La musique comme analyseur : mutations de la filière musicale et mutation de la recherche sur la musique

l'interassociation, Paris 22 mars 2004. Documentaliste, 41(2), 120-123.

Baudrillard, J. (1970). La société de consommation. Paris, France : Gallimard.

Becker, H.S. (1985). Outsiders: études de sociologie de la déviance. Paris, France : Métailié.

Becker, H. S. (2003). Paroles et musique : livre-disque. Paris, France : L'Harmattan.

Beuscart, J.-S., Coavoux, S. et Maillard, S. (2019). Les algorithmes de recommandation musicale et l'autonomie de l'auditeur: analyse des écoutes d'un panel d'utilisateurs de streaming. Réseaux, (213), 17-47. doi: 10.3917/res.213.0017

Boltanski, L. et Chiapello, E. (1999). Le nouvel esprit du capitalisme. Paris, France : Gallimard.

Boullier, D. (2009). Les industries de l'attention: fidélisation, alerte ou immersion. Réseaux, (154), 233-246.

Bourreau, M. et Gensollen, M. (2006). L'impact d'Internet et des technologies de l'information et de la communication sur l'industrie de la musique enregistrée. Revue d'économie industrielle, (116). https://doi.org/10.4000/rei.459

Bourreau, M. et Labarthe-Piol, B. (2004). Le peer to peer et la crise de l'industrie du disque. Une perspective historique. Réseaux, (125), 17-54.

Bourreau, M., Gensollen, M. et Moreau, F. (2007). Musique enregistrée et numérique : quels scénarios d'évolution de la filière ?. Culture prospective, 1(1), 1-16. doi: $10.3917 /$ culp.071.0001

Bourreau, M., Moreau, F. et Senellart, P. (2011). La diversité culturelle dans l'industrie de la musique enregistrée en France (2003-2008). Culture études, 5(5), 1-16. doi: 10.3917/cule.115.0001

Bourreau, M., Maillard, S. et Moreau, F. (2015). Une analyse économique du phénomène de la longue traîne dans les industries culturelles. Revue française d'économie, xxx(2), 179-216. https://doi.org/10.3917/rfe.152.0179 


\section{Christophe MAGIS et Lucien PERTICOZ}

Bouquillion, P. (2008). Les industries de la culture et de la communication. Les strategies du capitalisme. Grenoble, France : Presses universitaires de Grenoble.

Bouquillion, P. (2020). Les stratégies de visibilité, le rôle des plateformes. Enjeux numériques, (10), 21-26.

Bouquillion, P. et Combès, Y. (dir.) (2011). Diversité et industries culturelles. Paris, France : l'Harmattan.

Bouquillion, P. et Matthews, J. (2010). Le Web collaboratif: mutations des industries de la culture et de la communication. Grenoble, France : Presses universitaires de Grenoble.

Bouquillion, P., Miège, B. et Mœglin, P. (2013). L'industrialisation des biens symboliques. Les industries creatives en regard des industries culturelles. Grenoble, France : Presses universitaires de Grenoble.

Bruner, G. (1990). Music, mood and marketing. The Journal of Marketing, 54(4), 94-104.

Bull, M. (2000). Sounding Out the City. Personal Stereos and the Management of Everyday Life. Oxford, Royaume-Uni : Berg.

Bull, M. (2007). Sound Moves. iPod Culture and Urban Experience. Londres, Royaume-Uni : Routledge.

Bullich, V. (2011). Le droit d'auteur en regard de la théorie des industries culturelles. Les Enjeux de l'information et de la communication, (12/1), 51-68. doi: 10.3917/enic.011.0004

Cardon, D. (2018). Le pouvoir des algorithmes. Pouvoirs, (164), 63-73. doi: 10.3917/pouv.164.0063

Celma, O. et Cano, P. (2008). From hits to niches? Or how popular artists can bias music recommendation and discovery. Dans NETFLIX '08: Proceedings of the $2^{\text {nd }} K D D$ Workshop on Large-Scale Recommender Systems and the Netflix Prize Competition, article $\mathrm{n}^{\circ} 5$, 1-8. https://doi.org/10.1145/1722149 .1722154

Citton, Y. (dir.) (2014). L'économie de l'attention. Paris, France : La Découverte. 
La musique comme analyseur : mutations de la filière musicale et mutation de la recherche sur la musique

Creton, C. (2018). To pay or not to pay: les musiciens à notoriété locale face à la publicité ciblée sur Facebook. Les Enjeux de l'information et de la communication, 19(2), 15-28. doi: 10.3917/enic.025.0015

Curien, N. et Moreau, F. (2006). L'industrie du disque. Paris, France : La Découverte.

DeNora, T. (2000). Music in Everyday Life. Cambridge, Royaume-Uni : Cambridge University Press.

DeNora, T. (2001). Quand la musique de fond entre en action. Terrain, (37), 75-88. https://doi.org/10.4000/terrain.1310

Donnat, O. (2018). Évolution de la diversité consommée sur le marché de la musique enregistrée, 2007-2016. Culture études, 4(4), 1-32. doi: 10.3917/cule.184.0001

Doyle, G. (2002). Understanding Media Economics. Londres, Royaume-Uni/New York, NY : Sage.

Dumazedier, J. (1962). Vers une civilisation du loisir. Paris, France : Seuil.

Dupuy-Salle, M. (2019). La découverte musicale au prisme des dispositifs prescriptifs de Deezer et de leurs appropriations sociales. Dans V. Croissant (dir.), L'avis des autres: prescription et recommandation culturelles à l'ère numérique (p. 83-106). Paris, France: Éditions des Archives contemporaines.

Farchy, J., Méadel, C. et Anciaux, A. (2017). Une question de comportement. Recommandation des contenus audiovisuels et transformations numériques, ticetsociété, 10(2-3), 168198.

Faulkner, R. (1983). Music on demand. Composers and Careers in the Hollywood Music Industry. New Brunswick, NJ : Transaction Pub.

Flichy, P. (1997). Une histoire de la communication moderne. Espace public et vie privée, Paris, France : La Découverte.

Frith, S. (1996). Music and Identity. Dans S. Hall et P. Du Gay,(dir.), Questions of Cultural Identity (p. 108-127). Londres, Royaume-Uni : Sage.

Gayraud, A. et Heuguet, G. (2015). De l'industrie musicale à la rhétorique du "service ". YouTube : une description critique. 


\section{Christophe MAGIS et Lucien PERTICOZ}

Communication et langages, (184), 101-119. doi: $10.4074 / \mathrm{S} 0336150015012065$

George, É. (2014). The Theory of the Cultural Industries: A "Milieu" for Building Dynamic Knowledge. Canadian Journal of Communication, 39(1), 29-54.

Gilliotte, Q. (2019). L'expérience des biens culturels en régime numérique (Thèse de doctorat). Télécom Paris, Paris.

Gomez-Mejia, G., Nicey, J. et Vaezi, S. (2016). Questionner les pouvoirs éditoriaux de Google. Communication et langages, (188), 23-43. doi: 10.4074/S0336150016012023

Granjon, F. et Combes, C. (2007). La numérimorphose des pratiques de consommation musicale, Réseaux, (145-146), 291-334.

Granjon, F, Guyot, J. et Magis, C. (dir.) (2019). Matérialismes, culture et communication. T.3: Économie politique de la culture, des medias et de la communication. Paris, France : Presses des Mines.

Gras, S. É. (2014). L'écoute en ligne. Figures du sujet écoutant et mutations des espaces musicaux sur Internet (Thèse de doctorat). Université Paris 4, Paris.

Gras, S. É. (2015). Politique du streaming musical : discipline de l'écoutable et machines du goût. Poli, politiques de l'image, (11), 78-85.

Hamelink, C. J. (1983). Cultural Autonomy in Global Communications. Planning National Information Policy. New York, NY : Longman.

Hennion, A. (1981). Les professionnels du disque. Une sociologie des variétés. Paris, France : A.-M. Métailié.

Hennion, A. (1998). D'une distribution fâcheuse: analyse sociale pour les musiques populaires, analyse musicale pour les musiques savantes. Musurgia, 5(2), 9-19.

Hennion, A. (2004). Une sociologie des attachements: d'une sociologie de la culture à une pragmatique de l'amateur. Sociétés, (85), 9-24. doi: 10.3917/soc.085.0009

Hennion, A. (2007). La Passion musicale : une sociologie de la médiation. Paris, France: Éditions Métailié. doi: 10.3917/meta.henni.2007.01 
La musique comme analyseur : mutations de la filière musicale et mutation de la recherche sur la musique

Hennion, A. (2009). Réflexivités. L'activité de l'amateur. Réseaux, (153), 55-78. doi: 10.3917/res.153.0055

Hesmondhalgh, D. (2013). Cultural industries (3 édition). Londres, Royaume-Uni : Sage.

Horkheimer, M. et Adorno, T. W. (1974). La dialectique de la raison. Paris, France : Gallimard.

Huet, A., Ion, J., Lefèbvre, A., Miège, B., Péron, R. (1978). Capitalisme et industries culturelles. Grenoble, France: Presses universitaires de Grenoble.

IFPI (2020). Global Music Report - The Industry in 2019. Londres, Royaume-Uni : International Federation of the Phonographic Industry.

Lanham, R. (2006). The Economics of Attention. Style and Substance in the Age of Information, Chicago, IL : University of Chicago Press.Lanza, J. (2004). Elevator Music. Ann Arbor, MI : University of Michigan Press.

Le Guern, P. (2014). Réseaux et la culture: des médias traditionnels à la numérimorphose des goûts et des usages. Réseaux, (184-185), 211-246. doi: 10.3917/res.184.0211

Le Guern, P. (dir.) (2016). Où va la musique ? Numérimorphose et expériences d'écoute. Paris, France : Presses des Mines.

Magaudda, P. (2019). Infrastructures de la musique numérisée: les promesses de la «révolution» de la blockchain. Revue d'anthropologie des connaissances, 13(3), 849-869. doi: 10.3917/rac.044.0849

Magis, C. (2015). La musique et la publicité. Les logiques socioéconomiques et musicales des mutations des industries culturelles, Paris, France : Mare et Martin.

Magis, C. (2019). Bernard Miège. Une «théorie des industries culturelles " au sein des sciences de l'information et de la communication. Dans F. Granjon, J. Guyot et C. Magis (dir.), Matérialismes, culture et communication. T.3: Économie politique de la culture, des medias et de la communication (p. 136-160). Paris, France : Presses des Mines.

Magis, C. (2020). "Sur un air latino ». Le succès des tubes hispanophones dans la mondialisation. Hermès, La Revue, (86), 74-80. 


\section{Christophe MAGIS et Lucien PERTICOZ}

Maisonneuve, S. (2012). Techno-logies musicales. Communications, (91), 77-92. doi: 10.3917/commu.091.0077

Maisonneuve, S. (2019). L'économie de la découverte musicale à l'ère numérique : une révolution des pratiques amateurs ?. Réseaux, (213), 49-81. doi: 10.3917/res.213.0049

Mattelart, A. (2007). Diversité culturelle et mondialisation. Paris, France : La Découverte.

Mattelart, A., Delcourt, X. et Mattelart, M. (1983). La culture contre la démocratie ? L'audiovisuel à l'heure transnationale. Paris, France : La Découverte.

Mattelart, T. (2011). La "diversité culturelle »: quelques éléments de déconstruction théorique. Dans $P$. Bouquillion, et Y. Combès (dir.), Diversité et industries culturelles (p. 23-37). Paris, France : L'Harmattan.

Matthews, J. (2012). Prendre au sérieux l'industrie musicale. Dans J. Matthews et L. Perticoz (dir.), L'industrie musicale à l'aube du XXIe siècle. Approches critiques (p.7-18). Paris, France : L'Harmattan.

Matthews, J. (2015). Passé, présent et potentiel des plateformes collaboratives Réflexions sur la production culturelle et les dispositifs d'intermédiation numérique. Les Enjeux de l'information et de la communication, 16 (1), 5771. doi: $10.3917 /$ enic. 018.0057

Matthews, J. et Perticoz, L. (dir.) (2012). L'industrie musicale à l'aube $d u X X I^{e}$ siècle. Approches critiques. Paris, France: L'Harmattan.

Micheau, B., Després-Lonnet, M. et Cotte, D. (2017). La recommandation musicale entre inscriptions documentaires, pratiques sociales, et dispositifs d'écoute. Études de communication, 49(2), 33-56. https://doi.org/10.4000/edc.7014

Middleton, R. (1990). Studying Popular Music. Milton Keynes, Royaume-Uni : Open University Press.

Miège, B. (2007). La société conquise par la communication. T.3: Les Tic, entre innovation technique et ancrage social. Grenoble, France : Presses universitaires de Grenoble. 
La musique comme analyseur : mutations de la filière musicale et mutation de la recherche sur la musique

Miège, B. (2017). Les industries culturelles et créatives face à l'ordre de l'information et de la communication. Grenoble, France : Presses universitaires de Grenoble.

Moreau, F. et Peltier, S. (2004). Cultural Diversity in the Movie Industry: A Cross-National Study. Journal of Media Economics, 17(2), 123-143.

Morin, E. (1975). L'esprit du temps 1. Névrose. Paris, France : Grasset.

Morris, J. (2015a). Selling digital music, formatting culture. San Francisco, CA : University of California Press.

Morris, J. (2015b). Curation by code. Infomediaries and the data mining of taste. European Journal of Cultural Studies, 18(4-5), 446-463.

Nordenstreng, K., et Schiller, H. I. (dir.) (1979). National Sovereignty and International Communication. Norwood, NJ : Ablex.

Nowak, N. (2013). Consommer la musique à l'ère du numérique : vers une analyse des environnements sonores, Volume! La revue des musiques populaires, 10(1), 227-228. https://doi.org/10.4000/volume.3808

Nowak, N. (2016). Consuming Music in the Digital Age: Technologies, Roles and Everyday Life. Hampshire, Royaume-Uni : Palgrave Macmillan.

Ouellet, M. (2016). La révolution culturelle du capital. Le capitalisme cybernétique dans la société globale de l'information. Montréal, Canada : Les Éditions Ecosociété.

Perticoz, L. (2012a). La mise en musique du quotidien. Dans J. Matthews et L. Perticoz (dir.), L'industrie musicale à l'aube $d u \quad X X I^{e}$ siècle (p. 21-44). Approches critiques. Paris, France : L'Harmattan.

Perticoz, L. (2012b). Les industries culturelles en mutation : des modèles en question. Revue française des sciences de l'information et de la communication, https://doi.org/10.4000/rfsic. 112

Perticoz, L. (2016). Opulence musicale et recommandation : quelques réserves face à une "révolution » en cours. Dans P. Le Guern (dir.), Où va la musique ? Numérimorphose et 


\section{Christophe MAGIS et Lucien PERTICOZ}

nouvelles expériences d'écoute (p. 127-140). Paris, France: Presses des Mines.

Prey, R. (2018). Nothing personal: Algorithmic individuation on music streaming platforms. Media, Culture and Society, 40(7), 1086-1100.

Prior, N. (2012). Musiques populaires en régime numérique : acteurs, équipements, styles et pratiques. Réseaux, (172), 66-90. doi: 10.3917/res.172.0066

Rao, C. R. (1982). Diversity and dissimilarity coefficients: A unified approach. Theoretical Population Biology, 21(1), 24-43. https://doi.org/10.1016/0040-5809(82)90004-1

Roberge, J., Jamet, R. et Rousseau, A. (2019). L'impact social des algorithmes de recommandation sur la curation des contenus musicaux francophones au Québec. INRS - Institut national de la recherche scientifique (Centre Urbanisation Culture Société), Québec.

Rouzé, V. (2004). Les musiques diffusées dans les lieux publics : analyse et enjeux de pratiques communicationnelles quotidiennes (Thèse de doctorat). Université Paris 8, Paris.

Rouzé, V. (2005). Musicaliser le quotidien : analyse et enjeux de mises en scène particulières. Volume! La revue des musiques populaires. 4(2), 41-50. https://doi.org/10.4000/ volume. 1334

Rouzé, V. (2010). Mythologie de l'iPod. À l'écoute du temps présent. Paris, France : Éditions Cavalier Bleu.

Rouzé, V., Matthews, J. et Vachet, J. (2014). La Culture par les foules? Le crowdfunding et le crowdsourcing en question. Paris, France : MkF Éditions.

Rouzé, V. et Matthews, J. (2018). Les plateformes de crowdfunding culturel : entre figures de l'artiste entrepreneur et entrepreneurs polymorphes. Les Enjeux de l'information et de la communication, 19(1), 35-50. doi: 10.3917/enic.024.0035

Scherzinger, M. (2005). Music, corporate power and unending war. Cultural Critique, 60(1), 27-63. 
La musique comme analyseur : mutations de la filière musicale et mutation de la recherche sur la musique

Schöpfel, J. (2006). The new French law on author's right and related rights in the information society. Interlending and Document Supply, 34(4), doi: 10.1108/02641610610714740

Seaver, N. (2018). Captivating algorithms: Recommender systems as traps. Journal of Material Culture, 24(4), 421436.

Shuker, R. (2008). Understanding Popular Music Culture, Londres, Royaume-Uni/New York, NY : Routledge.

Smyrnaios, N. (2017). Les GAFAM contre l'internet. Une économie politique du numérique. Bry-sur-Marne, France: INA Éditions.

SNEP. (2020). La production musicale française 2019. Paris, France : Syndicat national de l'édition phonographique.

Sterne, J. (2012). MP3: The meaning of a format. Durham, NC : Duke University Press.

Sterne, J. (2015). Le MP3 comme artefact culturel. Communication et langages, (184), 41-60. doi: $10.4074 / S 033615001501203 X$

Sterne, J., Sklower, J. et Heuguet, G. (2017). Du charivari au big data. Les musiques populaires au prisme des sound studies : entretien avec Jonathan Sterne. Volume ! La revue des musiques populaires, 14(1), 175-192.

Stirling, A. (1998). On the economics and analysis of diversity. SPRU Electronic Working Paper, (28).

Szendy, P. (2001). Écoute. Une histoire de nos oreilles. Paris, France : Éditions de Minuit.

Tournès, L. (2011). Musique ! Du phonographe au MP3 (18772011) ( $2^{\mathrm{e}}$ édition). Paris, France : Autrement.

Tremblay, G. (2007). Espace public et mutations des industries de la culture et de la communication. Dans P. Bouquillion, $\mathrm{P}$. et Combès, Y. (dir.), Les industries de la culture et de la communication en mutation (p. 207-225). Paris, France: L'Harmattan.

Truax, B. (1978). Handbook for Acoustic Ecology. Vancouver, Canada : ARC Publications. 


\section{Christophe MAGIS et Lucien PERTICOZ}

Truax, B. (1985). Acoustic Communication. Norwood, NJ: Ablex.

Turner, F. (2012). Aux sources de l'utopie numérique. De la contre-culture à la cyberculture: Stewart Brand un homme d'influence. Caen, France : C\&F Éditions.

Vandiedonck, D. (2007). L'industrie de la musique enregistrée recomposée. Dans P. Bouquillion et Y. Combès (dir.), Les industries de la culture et de la communication en mutation (p. 91-98). Paris, France : L'Harmattan.

Vercellone, C. (2020). Les plateformes de la gratuité marchande et la controverse autour du Free Digital Labor: une nouvelle forme d'exploitation?. Revue ouverte d'ingénierie des systèmes d'information, 20(2). doi: 10.21494/ISTE.OP.2020.0502

Williamson, J. et Cloonan, M. (2007). Rethinking the music industry. Popular Music, 26(2), 305-322. 